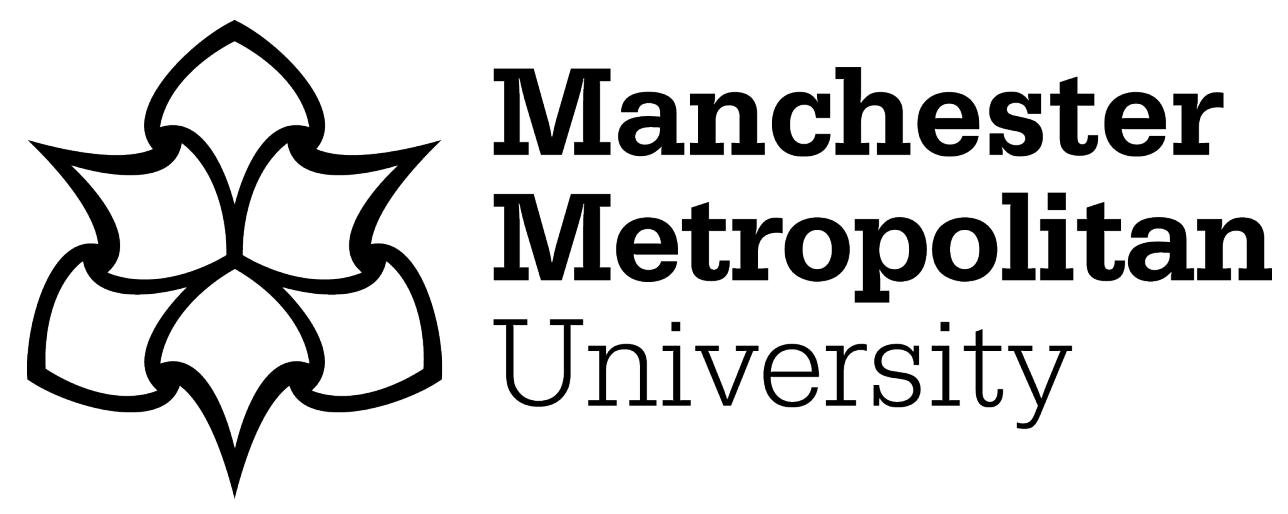

Jiang, Sheng-Chao and Bai, Wei ORCID logoORCID: https://orcid.org/00000002-3537-207X (2020) Coupling analysis for sway motion box with internal liquid sloshing under wave actions. Physics of Fluids, 32. 072106-072106. ISSN 1070-6631

Downloaded from: https://e-space.mmu.ac.uk/626220/

Version: Accepted Version

Publisher: AIP Publishing

DOI: https://doi.org/10.1063/5.0015058

Please cite the published version 


\title{
Coupling analysis for sway motion box with internal liquid sloshing under wave actions
}

\author{
Sheng-chao Jiang (姜胜超) 1,2 , a) and Wei Bai (柏威 $)^{3}$ \\ ${ }^{1)}$ School of Naval Architecture, State Key Laboratory of Structural Analysis for Industrial Equipment, \\ Dalian University of Technology, Dalian 116024, China \\ ${ }^{2)}$ State Key Laboratory of Coastal and Offshore Engineering, Dalian University of Technology, Dalian 116024, \\ China \\ ${ }^{3)}$ Department of Computing and Mathematics, Manchester Metropolitan University, Chester Street, Manchester M1 5GD, \\ $U K$
}

(Dated: 4 July 2020)

\begin{abstract}
This work investigates the coupling effects of internal sloshing flow on the sway motion response of rectangular box sections. The impulse-response-function (IRF) method is employed for external wave action, while the viscous twophase flow model with the volume of fluid (VOF) interface capturing technique based on the OpenFOAM ${ }^{\circledR}$ package is adopted for the internal sloshing flow. A new lower critical frequency is defined to understand the coupling effects of the internal sloshing flow, which is the corresponding frequency of the minimal sway motion amplitude. The external wave and internal sloshing forces are out-of-phase at the lower critical frequency. The numerical simulations show that the lower critical frequency is equivalent to the sloshing natural frequency when the internal sloshing flow is in the nonbreaking pattern. The non-breaking sloshing-induced force approaches the same magnitude as the external wave force, which leads to a zero-amplitude sway motion. When the internal sloshing exhibits the breaking phenomenon, a phase transition of the internal sloshing force can occur, which causes the lower critical frequency to be smaller than the sloshing natural frequency. The increased incident wave amplitude or decreased tank breadth can strengthen the nonlinear behavior of the sloshing coupling action. That is, the sway motion response deviates more from the linear sloshing flow results, including the smaller lower critical frequency and the larger minimal sway motion amplitude. However, with the increased breaking-sloshing-induced nonlinearity, the difference in the sway motion response between the coupling and uncoupling results reduces, which implies a lower coupling effect.
\end{abstract}

\section{INTRODUCTION}

As the increased demand for liquefied natural gas (LNG) and liquefied petroleum gas (LPG), floating production storage and offloading (FPSO) and floating liquefied natural gas (FLNG) production systems have attracted significant recen$\mathrm{t}$ attention. These structures possess ship-type floating hulls for operation and production, and the liquefaction plants and liquid tanks are equipped for LNG or LPG storage. When the storage tanks are partially filled, the ship motion response is affected not only by the external wave excitation but also by the internal liquid sloshing. The interaction reflects the coupling effect between the ship motion and the internal sloshing under wave actions, which leads to dramatic variations in the ship motion response. A similar phenomenon is found in loading or offloading operations of LNG carriers or shuttle tankers. Therefore, a better understanding of the coupling effects between the liquid sloshing and ship motion response has broad engineering applications for the safety of offshore exploration and marine operations.

Early studies on the coupling sloshing problem were performed in the framework of a linear potential flow model and classic diffraction theory in the frequency domain, such as Molin (2002), Malenica, Zalar, and Chen (2003), Newman (2005), and Gou, Kim, and Kim (2011). In these works, the sloshing-induced force was decomposed into the restoring force and added mass, where the coupling effects of the

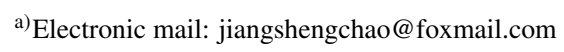

internal sloshing flow on the ship motion response were considered in the ship motion equation. However, the linear potential flow model is not suitable to simulate violent internal sloshing flow motion. This has been demonstrated by extensive comparisons of the ship motion response between linear potential flow solutions and experimental measurements. With the development of computing technologies and numerical techniques, the numerical wave flume model in the time domain has been taken as an alternative method for coupling sloshing problems. Wang, Sun, and $\mathrm{Hu}$ (2017) established a two-dimensional fully-nonlinear numerical approach using the boundary element method (BEM). The coupling of the floating tank motion with the nonlinear internal sloshing flow under the fifth-order Stokes wave action was investigated. Zhuang and Wan (2019) studied ship motion with the sloshing effect in waves using a full computational fluid dynamics (CFD) simulation. Cao et al. (2019) conducted a coupled analysis of a damaged ship with internal sloshing in beam seas, where a numerical wave flume based on the smooth particle hydrodynamics (SPH) method was adopted. These investigations confirm that the sloshing flow nonlinearity can significantly affect the behavior of ship motion responses.

For an increased efficiency compared to the full numerical wave flume method, a hybrid approach that combines the external wave action and internal sloshing flow motion has been developed. Mitra et al. (2012) developed a weak-form Galerkin finite element method (FEM) to solve the internal sloshing flow, while the sea-keeping problem was simulated with a hybrid marine control system. Cercos-Pita et al. (2016) and Serván-Camas et al. (2016) adopted a nonlinear 
ship motion program to determine the response with six degrees of freedom, and used the SPH method for the internal sloshing motion. The essential advantage of the hybrid numerical model is that suitable methods can be adopted in the external wave action and internal sloshing flow, respectively. As is well known, ship motion is a classical marine hydrodynamic problem that can be accurately simulated using the linear potential flow model. On the other hand, the liquid sloshing problem represents one kind of complex interface flow with a highly nonlinear breaking phenomenon, which requires more advanced numerical models. Using this principle, Kim 2002) recommended the linear potential flow model with a combined Rankine source and transient Green-function method for the external wave action and the Eulerian equation for the internal sloshing action. Huang et al. (2018) and Saripilli and Sen (2018) applied the time-domain transient Greenfunction method to the ship motion response, and the internal sloshing flow was simulated with the nonlinear potential flow model equipped with artificial damping and a Navier-Stokes solver, respectively.

The impulse-response function (IRF) method (Cummins, 1962 ) is another popular approach for the time-domain analysis of ship motion in coupling sloshing problems. The IRF method is built on top of the frequency-domain solution$\mathrm{s}$ for the diffraction/radiation potential flow model; thus, it can provide a higher computing efficiency than direct timedomain solvers. Therefore, more computational resources can be used for internal sloshing flow simulations. Rognebakke and Faltinsen (2003) adopted the multimodal method to simulate the internal sloshing flow motion to investigate the coupling effects on the sway motion box section. Nam et al. (2009) simulated liquid sloshing using the finite difference method (FDM) based on the Eulerian equation. The numerical study covered three degrees of freedom (heave, pitch, and rol1) of a simplified LNG/FPSO unit. Zhao et al. (2014) and $\mathrm{Su}$ and Liu (2017) adopted the nonlinear potential flow model and the nonlinear Boussinesq-type approach for the liquid sloshing problem to address the nonlinear coupling action around the sloshing natural frequency, respectively. Zhao et al. (2018) conducted a nonlinear potential flow simulation for the coupled sloshing action on a barge vessel with six degrees of freedom. The most significant coupling action was observed in the sway and roll motions for beam seas, while the surge and pitch motions in the head seas were less sensitive to the internal sloshing flow action. Li et al. (2019) simulated the influence of liquid sloshing on the motion response of a ship with forward speeds, where the increasing trend of the coupling effect on the pitch motion can be observed with an increased forward speed. These works confirm that the free surface nonlinearity in liquid sloshing significantly impacts the coupling behavior of ship motion response. However, the inviscous fluid flow model cannot simulate the damping effect during the coupled sloshing process.

To better understand the damping effect on the coupled interaction between the ship motion and internal sloshing, Rognebakke and Faltinsen (2003) adopted a linear artificial damping term in the sloshing flow model. The calculated coupled sway motion response is sensitive to the damping of the sloshing motion around its natural frequency. Lee and Kim (2010) adopted the Navier-Stokes solver for the internal sloshing motion, where both the fluid viscosity and free surface nonlinearity are considered in the coupling action. However, these sloshing flow models adopted a single-valued function for the free surface motion, which implies the influence of complex interface flows, such as folding, breaking, spraying, and overturning, cannot be simulated correctly. Jiang et al. (2015) simulated the internal sloshing flow using the NavierStokes solver with the volume of fluid (VOF) method. The numerical simulations showed that the sloshing flow configuration significantly affects the rolling motion response, which leads to nonlinear variations in the ship rolling RAOs with the incident wave amplitude, especially around the ship and sloshing motion natural frequencies. Du, Wang, and Zhang (2019) adopted the Navier-Stokes solver with the multi-material arbitrary Lagrangian-Eulerian (MMALE) method to remap the broken surface of the sloshing motion. The spraying phenomenon was observed under large amplitude incident waves around the sloshing natural frequency, where the normalized sway motion amplitude is sensitive to the incident wave amplitude. In the coupling sloshing system, the sloshing action is considered as a frequency-dependent spring, where the damping that involves the breaking phenomenon can significantly impact the spring stiffness. This is why the sloshing coupling action has amplitude-dependent characteristics at various frequencies. Although these research efforts demonstrate the significance of internal sloshing flow patterns, detailed variations in the ship motion response with the state of the internal sloshing flow motion still lack comprehensive numerical investigations, especially involving breaking phenomena. As is well known, the internal sloshing flow motion has different characteristics before and after breaking (Bouscasse et al. 2014a b; Sauret et al. 2015, Lu et al., 2015). Therefore, significant differences in the ship motion responses between non-breaking and breaking sloshing configurations are expected, which is the primary motivation of this study.

The behavior of the sway motion response with the coupling of the internal sloshing flow motion is investigated based on the above issues. A series of numerical simulations are performed for the sway motion box section with different tank breadths and filling depths under wave actions. As the violent free surface motion is reported as a concern in sloshing flow motions, the numerical investigations focus on the influence of the sloshing flow configurations on the sway motion response. Differences in the sway motion responses as affected by the non-breaking and breaking sloshing flow motions are identified, and a new concept of the lower critical frequency is defined to describe its characteristics. Three patterns for the sway motion response with the coupling sloshing action are addressed. Thus, numerical analyses are performed to investigate various influencing factors in this study, and some dynamic mechanisms of the coupling sloshing are revealed. 


\section{MATHEMATICAL FORMULATION}

A rectangular sway box section equipped with a partiallyfilled tank is considered, as shown in Fig. 1. Two Cartesian coordinates are defined in the figure, with $O X Y Z$ as the spacefixed system (simulates the external wave motion and the coupling strategy) and oxyz as the body-fixed system (simulate the internal sloshing flow motion). A brief description of the hybrid numerical model is described as follows.

\section{A. Internal liquid sloshing flow model}

The Navier-Stokes equations for incompressible two-phase flow in an arbitrary Lagrangian-Eulerian (ALE) reference system is adopted here to describe the internal sloshing flow,

$$
\begin{gathered}
\frac{\partial \rho}{\partial t}+\frac{\partial \rho u_{i}}{\partial x_{i}}=0 \\
\frac{\partial \rho u_{i}}{\partial t}+\frac{\partial \rho\left(u_{j}-u_{j}^{m}\right) u_{i}}{\partial x_{j}}=\rho g_{i}-\frac{\partial p}{\partial x_{i}} \\
+\mu \frac{\partial}{\partial x_{j}}\left(\frac{\partial u_{i}}{\partial x_{j}}+\frac{\partial u_{j}}{\partial x_{i}}\right)
\end{gathered}
$$

where $u_{i}$ is the velocity component in the $i$ th direction, $u_{i}^{m}$ is the velocity component due to the mesh deformation in the ALE frame, $p, \rho$, and $g_{i}$ are the pressure, fluid density, and gravitational acceleration, respectively, and $\mu$ is the fluid dynamic viscosity.

To simulate the violent sloshing flow, especially the folding, breaking, and overturning phenomena, the volume of fluid (VOF) method (Hirt and Nichols, 1981) is adopted to capture the free surface motion. The fractional function of the VOF, as defined by $\varphi$, in a computational cell is given by,

$$
\varphi= \begin{cases}0, & \text { in air } \\ 0<\varphi<1, & \text { on free surface } \\ 1, & \text { in water }\end{cases}
$$

The fractional function satisfies the following advection equation,

$$
\frac{\partial \varphi}{\partial t}+\left(u_{i}-u_{i}^{m}\right) \frac{\partial \varphi}{\partial x_{i}}=0
$$

where the contour of the fractional function with $\varphi=0.5$ is used to represent the interface between the water and air phases. In the computation, the fluid density and viscosity are averaged using the available fractional function,

$$
\begin{aligned}
& \rho=\varphi \rho_{W}+(1-\varphi) \rho_{A}, \\
& \mu=\varphi \mu_{W}+(1-\varphi) \mu_{A},
\end{aligned}
$$

where the subscripts $W$ and $A$ represent the water and air phases, respectively.
The governing equations $(1 \mathrm{a})$ and $(1 \mathrm{~b})$ and the VOF equation (3) are solved using the finite volume method (FVM) integrated in the OpenFOAM ${ }^{\circledR}$ package. The velocity and pressure are implicitly decoupled from the pressure using the splitting of operators (PISO) algorithm (Issa, 1986), and the Euler method is used to discretize the transient term. The convection and diffusion terms are discretized using the Gauss limited linear method and Gauss linear corrected method, respectively. The no-slip boundary condition is imposed at the solid walls, which includes the body surface and seabed. The numerical computations always begin from the still state, which mean$\mathrm{s}$ the hydrostatic pressure and zero velocity are specified as the initial conditions. In the numerical simulations, the time increment is automatically determined based on the CourantFriedrichs-Lewy (CFL) condition as,

$$
\Delta t \leq C_{r} \times \min \left\{\sqrt{S_{e}} /\left|u_{e}\right|\right\}
$$

where $S_{e}$ and $\left|u_{e}\right|$ are the area and absolute velocity in the computational cell, respectively. The numerical results confirm that the coefficient of $C_{r}=0.20$ can produce stable and accurate results. For the details of the numerical implements in the OpenFOAM ${ }^{\circledR}$, the readers are referred to Jasak (1996) and Rusche (2003). Finally, the fluid force on the tank is obtained by integrating the pressure and shear stress over the body surface as,

$$
F(t)=-\int_{S} p \cdot \mathbf{n} d S+\int_{S} \mu \frac{\partial \vec{u}_{\tau}}{\partial n} d S
$$

where $S$ is the tank wall surface, and $\mathbf{n}$ and $\vec{u}_{\tau}$ are the unit normal vectors and the tangent velocity component, respectively.

\section{B. External wave action and coupling strategy}

The potential flow model with an empirical nonlinear drag formulation is utilized to simulate the external wave action. A convolution integral in the equation for box motion helps express the sway motion response $\zeta_{2}(t)$ in the time domain as,

$$
\begin{array}{r}
{\left[M_{b}+a_{22}(\infty)\right] \ddot{\zeta}_{2}(t)+B_{22}^{v i s} \dot{\zeta}_{2}(t)\left|\dot{\zeta}_{2}(t)\right|+} \\
\int_{0}^{t} R_{22}(t-\tau) \dot{\zeta}_{2}(\tau) d \tau= \\
F_{2}^{\text {ext }}(t)+F_{2}^{\text {int }}(t) \\
+F_{2}^{\text {bear }} \frac{\dot{\zeta}_{2}(t)}{\left|\dot{\zeta}_{2}(t)\right|}
\end{array}
$$

where $M_{b}$ is the box mass (excluding the fluid mass inside the box as this is included in $F_{2}^{\text {int }}$ ). The $F_{2}^{\text {bear }}$ is the constant force acting against the motion from the bearings, which is $2.0 \mathrm{~N}$ based on the physical experiments in Rognebakke and Faltinsen (2003). The $B_{22}^{\text {vis }}$ is the viscous damping coefficient due to the external fluid motion, which is estimated based on the assumption of a low Keulegan-Carpenter number flow,

$$
B_{22}^{v i s}=C_{D} \frac{\rho W d}{2}
$$




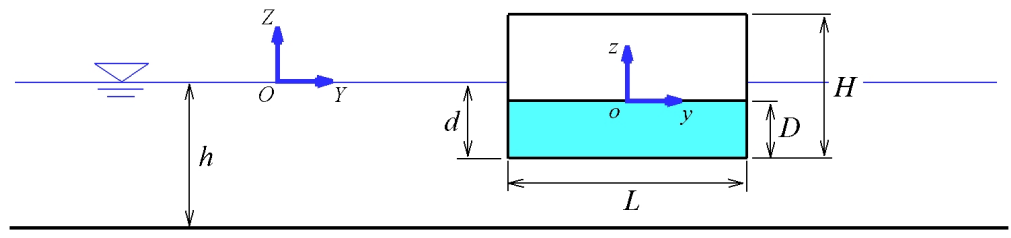

(a)

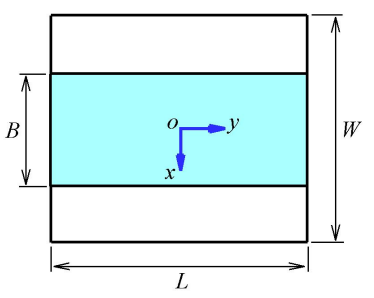

(b)

FIG. 1: Sketch of the coordinate definitions and the geometry of the sway box section. (a) Side view, (b) Top view.

where $W$ and $d$ are the width and draft of the sway box section, respectively, and $C_{D}$ is the drag coefficient valued at 0.30 for the present facing square problem. The $F_{2}^{i n t}$ is the internal liquid force along the sway direction and includes the liquid hydrostatic force and sloshing-induced force, which is calculated with Eq. (6) in Sec. II A The $F_{2}^{e x t}(t), a_{22}(\infty)$, and $R_{22}(t)$ are the exciting force, infinite frequency added mass, and the retardation function, respectively.

A two-dimensional classical potential flow model with the higher-order boundary element method (HOBEM) (Teng and Eatock Taylor, 1995) is adopted to compute the exciting forces $H_{i}(\omega)$, added mass $a_{i j}(\omega)$, and radiation damping $b_{i j}(\omega)$ in the frequency domain. Based on these results, the external hydrodynamic loads $F_{2}^{\text {ext }}(t), a_{22}(\infty)$, and $R_{22}(t)$ are obtained in the time domain using the impulse response function (IRF) method. For the radiation force, the relationship between the time and frequency domains can provide the added mass $a_{i j}(\omega)$ and radiation damping $b_{i j}(\omega)$ in terms of the Fourier transforms of the IRF as

$$
\begin{gathered}
b_{i j}(\omega)=\int_{0}^{\infty} R_{i j}(t) \cos (\omega t) d t \\
a_{i j}(\infty)=a_{i j}(\omega)+\frac{1}{\omega} \int_{0}^{\infty} R_{i j}(t) \sin (\omega t) d t .
\end{gathered}
$$

Furthermore, the inverse-transform of Eq. 9a can give an alternative expression for the IRF,

$$
R_{i j}^{e x t}(t)=\frac{2}{\pi} \int_{0}^{\infty} b_{i j}(\omega) \cos (\omega t) d \omega
$$

A similar relation also exists for the exciting force, while the time-domain external exciting force $F_{i}^{\text {ext }}(t)$ on the box is computed as

$$
F_{i}^{e x t}(t)=\int_{0}^{t} h_{i}(t-\tau) \eta(\tau) d \tau
$$

where $\eta(t)$ is the incident wave elevation, and $h_{i}(t)$ is the impulsive response function resulting from a unit impulse input, which can be obtained from the following IRF Fourier transforms,

$$
h_{i}(t)=\Re\left[\frac{1}{\pi} \int_{0}^{\infty} H_{i}(\omega) \mathrm{e}^{-\mathrm{i} \omega t} d t\right]
$$

Figure 2 shows the coupling strategy for the presented numerical model, which is applied using Eq. (7). There are three parts in the coupling model: external wave action, internal sloshing flow, and coupling analysis. The time-domain external wave action can be expressed with an impulse response function $h_{i}(t)$, added mass $a_{i j}(\infty)$, and retardation function $R_{i j}(t)$, which are computed by Eqs. (12), (9b). and (9a), respectively. When solving the motion response of Eq. (7), the internal and external fluid forces are computed using Eqs. (6) and (11), respectively. After solving Eq. (11) at the curren$\mathrm{t}$ time step, the motion response is obtained and adopted as the moving-wall boundary conditions for the simulation of the tank sloshing at the following time step. In the numerical simulation, the time step for the internal sloshing force is given by Eq. (5), and the box motion response can be solved at the same time step. For the external wave action, a B-spline interpolation scheme is required in both the wave excitation force $F_{i}^{e x t}(t)$ and the retardation function $R_{i j}(t)$. It is noted that 50 frequencies are employed in the frequency-domain computation for the convolution of the impulse response and excitation, and convergence of the computational solutions is ensured.

\section{NUMERICAL TEST AND VALIDATION}

Numerical investigations are performed for the sway motion response of the box section coupling with different internal filling conditions under the wave actions. Figure 1 shows the definition of the geometries for the wave flume and the sway box section, where the box section of length $L=0.376$ m, breadth $W=0.599 \mathrm{~m}$, height $H=0.40 \mathrm{~m}$, and draft $d=0.20$ $\mathrm{m}$ is located in the wave flume with a water depth $h=1.030 \mathrm{~m}$. Several simulation cases are designed to perform the intended investigation by varying the values of the tank breadth $B$ and filling depth $D$. The definition of the test cases is given in Table[1, where three tank breadths $B=0.150,0.225$, and $0.300 \mathrm{~m}$ and three filling depths $D=0.094,0.186$, and $0.290 \mathrm{~m}$ are selected together with the corresponding case of the zero-filling condition (indicated as Emptytank in the table). Numerical simulations follow the experiments in Rognebakke and Faltinsen (2003), in which the total mass (body mass plus the water mass $M_{b}+M_{w}$ ) is kept constant for different filling conditions. Four incident wave amplitudes, $A_{i}=0.005,0.010,0.020$, and $0.030 \mathrm{~m}$, are adopted, while the wave frequency $\omega$ varies 


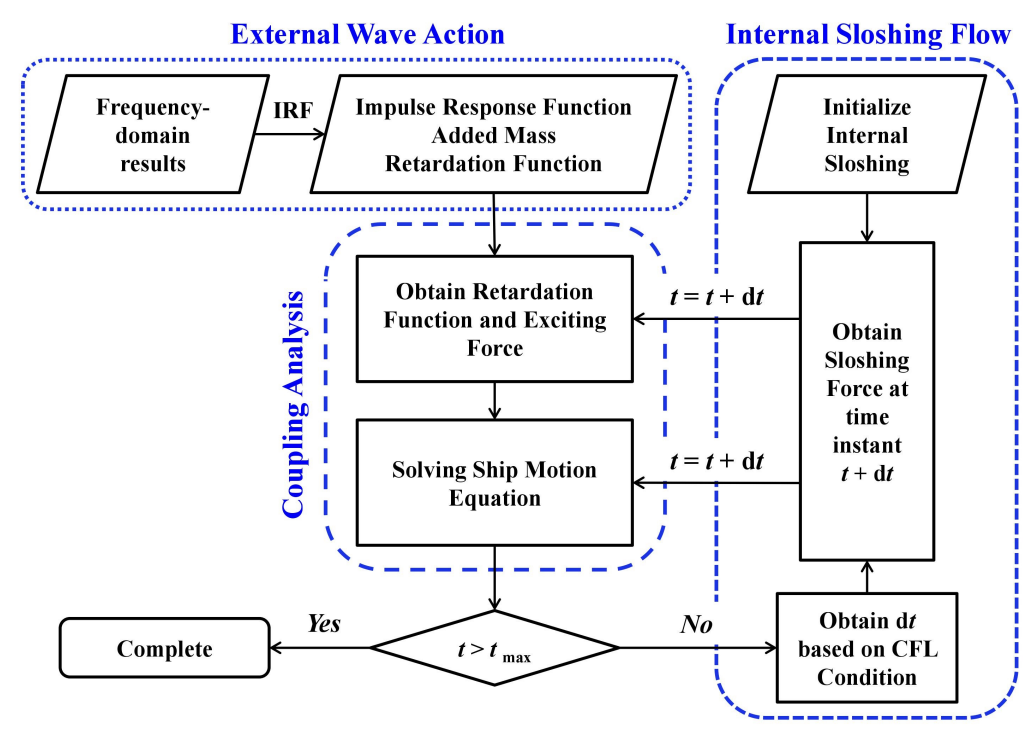

FIG. 2: Coupling strategy for the ship motion response with internal sloshing flow under wave action.

from 6.0-11.0 rad/s, which covers the natural frequency of the internal sloshing flow. To avoid initial transients, a cosine ramp function is adopted to modulate the exciting force over the initial 10 wave periods in the simulations.

Figure 3 shows three typical time signals for the sway motion responses, where the corresponding geometry and wave frequency are illustrated in the captions. It is observed from Fig. 3a that the amplitude of the sway motion response increases gradually at the initial stage before $t=17.5 \mathrm{~s}$. After a short transient, a steady-state evolution is observed in the sway motion signal, which is relatively symmetric as a sinusoidal function. The steady state of the sway motion response is sustained until the end of the computation at $100 \mathrm{~s}$, indicating that the coupling effects of the internal sloshing flow were fully developed at the 'steady-state stage.' A similar phenomenon is observed in Fig. 3b, however, a small transient appears again after the 'steady-state stage' at $t=38.5 \mathrm{~s}$. This is explained by the development of impact loading due to the violent broken-free surface motion around the resonant frequency with time. Fourier analysis indicates that the dominating harmonic of the sway motions is still at the incident wave frequency $\omega$ for the new transient evolution. The average amplitudes of the sway motion responses between the durations of $25-35$ and $50-100 \mathrm{~s}$ are $\overline{A_{2}} / A_{i}=0.469$ and 0.446 , respectively, while the maximal amplitudes are $A_{2}^{\max } / A_{i}=0.471$ and 0.475 , respectively. In general, only a slight discrepancy in the results between the steady state and new transient is observed, suggesting the new transient can be regarded as the 'near steady-state stage.' For the third type of evolution in Fig. 3c, only the 'initial stage' and 'near steady-state stage' are observed. Thus, the 'steady-state stage' disappears, implying that the impact loading from the violent sloshing flow develops more rapidly. In the following simulations, the sway motion amplitudes $A_{2}$ are computed from the averaged values over the 'near steady-state stage.' If the 'near steady-state stage' does not appear, the sway motion amplitude can be eas- ily determined from the 'steady-state stage.' Finally, it is noted that the evolutions in Fig. 3 are simulated with Mesh 3, a convergent mesh scheme, as discussed next.

A mesh dependency test is performed for the sway motion response using an incident wave amplitude of $A_{i}=0.015 \mathrm{~m}$ for three different geometries: B150D186, B150D094, and B150D290. Four sets of mesh schemes are adopted in the numerical tests, as detailed in Table II Non-uniform meshes are utilized to discretize the internal fluid domain in the CFD simulations to reduce the computational costs. Highresolution square fine meshes are adopted near the free surface, while intermediate-resolution rectangular meshes are used elsewhere. Typical meshes are depicted in Fig. 4, showing Mesh 1 in Table $\mathrm{II}$ for the three geometries. Figure 5 shows the amplitudes of the sway motion response obtained for the different mesh schemes. The numerical results suggest that the variations in the mesh density have little impact on the results when the number of cells is larger than $4 \times 10^{4}$, which confirms that Mesh 3 can produce convergent solutions. Therefore, Mesh 3 is adopted as the baseline for the following investigations.

An available experiment in Rognebakke and Faltinsen (2003) performed at the Norwegian University of Science and Technology is adopted to validate the accuracy of the numerical model, where the B150D186, B150D094, B150D290, and B300D186 geometries are considered. The relationship between the wave amplitude and the wave frequency is given in Eq. 13 as,

$$
A_{i}= \begin{cases}0.030, & 6.00 \leq \omega \leq 6.90 \\ 0.020, & 7.00 \leq \omega \leq 7.90 \\ 0.015, & 8.00 \leq \omega \leq 11.00\end{cases}
$$

Besides the laboratory measurements, the numerical results in Rognebakke and Faltinsen (2003) are also included for comparison. In their work, the numerical model was established 
TABLE I: List of test cases and corresponding geometries and parameters in the present study.

\begin{tabular}{cccccc}
\hline \multirow{2}{*}{ Name } & $\begin{array}{c}\text { Tank Breadth Filling Depth Body Mass } \\
(B / \mathrm{m})\end{array}$ & $\begin{array}{c}(D / \mathrm{m}) \\
\left(M_{b} / \mathrm{kg}\right)\end{array}$ & $\begin{array}{c}\text { Water Mass } \\
\left(M_{w} / \mathrm{kg}\right)\end{array}$ & $\begin{array}{c}\text { Natural Frequency } \\
\left(\omega_{n} / \mathrm{rad} / \mathrm{s}\right)\end{array}$ \\
\hline Emptytank & - & - & 47.01 & 0.00 & - \\
\hline B150D094 & 0.150 & 0.094 & 43.51 & 5.30 & 7.33 \\
B150D186 & 0.150 & 0.186 & 37.01 & 10.49 & 8.66 \\
B150D290 & 0.150 & 0.290 & 31.51 & 16.36 & 8.98 \\
\hline B225D094 & 0.225 & 0.094 & 40.86 & 7.95 & 7.33 \\
B225D186 & 0.225 & 0.186 & 31.76 & 15.74 & 8.66 \\
B225D290 & 0.225 & 0.290 & 23.33 & 24.53 & 8.98 \\
\hline B300D094 & 0.300 & 0.094 & 38.21 & 10.60 & 7.33 \\
B300D186 & 0.300 & 0.186 & 26.52 & 20.98 & 8.66 \\
B300D290 & 0.300 & 0.290 & 15.15 & 32.71 & 8.98 \\
\hline
\end{tabular}

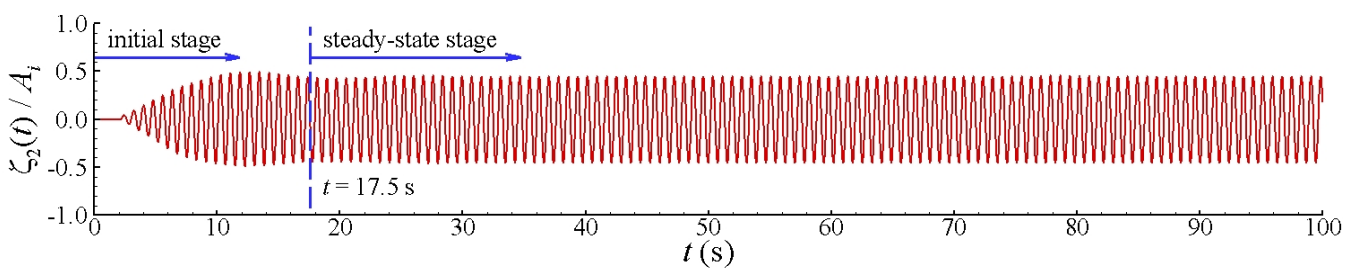

(a)

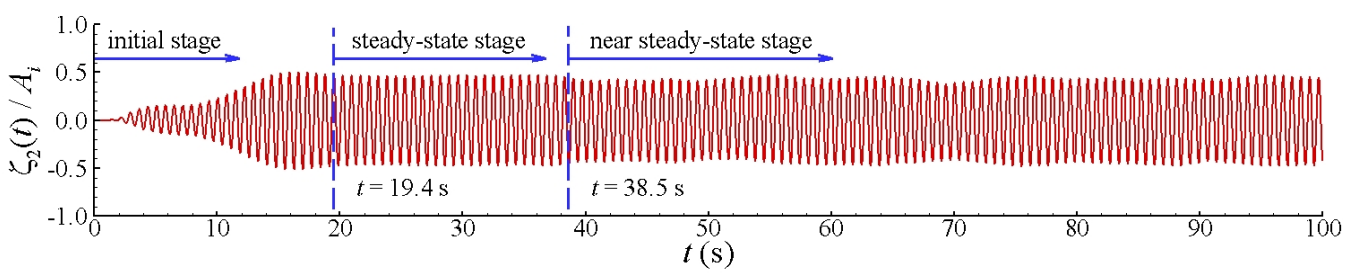

(b)

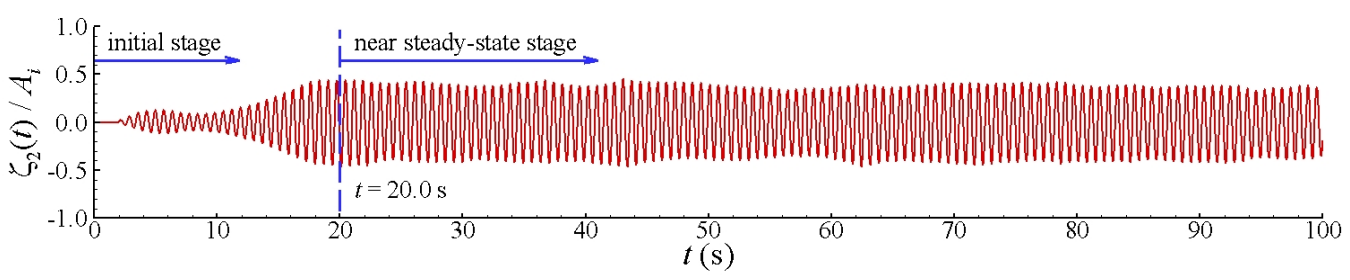

(c)

FIG. 3: Time signals of the sway motion response for the box under wave actions.
(a) $D=0.094 \mathrm{~m}, B=0.150 \mathrm{~m}, A_{i}=0.015 \mathrm{~m}$, and $\omega=8.00 \mathrm{rad} / \mathrm{s}$,
(b) $D=0.186 \mathrm{~m}, B=0.150 \mathrm{~m}, A_{i}=0.015 \mathrm{~m}$, and $\omega=8.70 \mathrm{rad} / \mathrm{s}$,
(c) $D=0.290 \mathrm{~m}, B=0.150 \mathrm{~m}, A_{i}=0.015 \mathrm{~m}$, and $\omega=8.90 \mathrm{rad} / \mathrm{s}$.

TABLE II: Mesh resolutions (number of cells in the computational domain) for the convergent tests.

\begin{tabular}{cccc}
\hline Name & $D=0.186 \mathrm{~m}$ & $D=0.094 \mathrm{~m}$ & $D=0.290 \mathrm{~m}$ \\
\hline Mesh 1 & 10434 & 12314 & 12500 \\
Mesh 2 & 23688 & 27777 & 28200 \\
Mesh 3 & 41924 & 49256 & 50000 \\
Mesh 4 & 95880 & 110826 & 105000 \\
\hline
\end{tabular}

using the IRF method for the external wave action and sway motion response, while a linear model without artificial damping and nonlinear model with artificial damping were adopted to simulate the internal sloshing flow. As shown in Fig. 6, the overall comparison suggests that the present viscous sloshing flow results and the nonlinear sloshing numerical results in Rognebakke and Faltinsen (2003) agree well with the experimental data. The numerical results at the non-resonant conditions agree better with the experiments than those at the resonant condition. Around the resonant frequency, the violent 


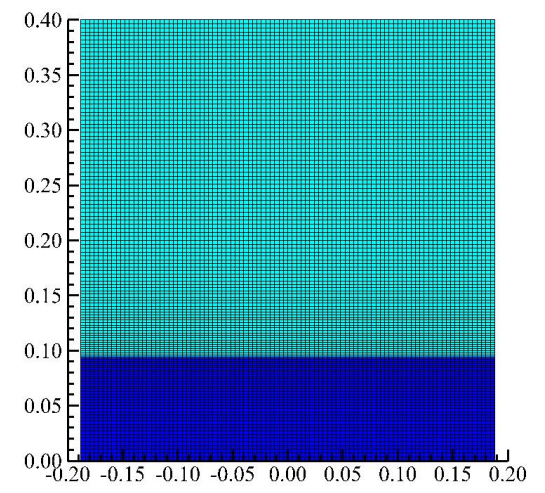

(a)

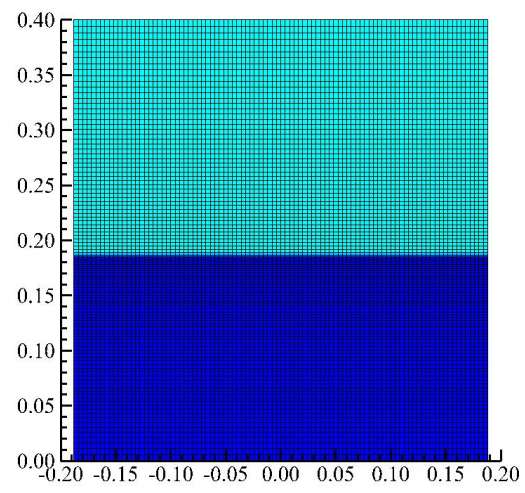

(b)

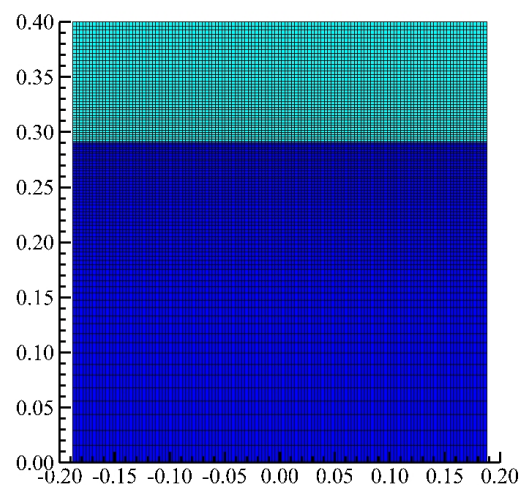

(c)

FIG. 4: Typical grid systems in the tank for different filling conditions. (a) $D=0.094 \mathrm{~m}$, (b) $D=0.186 \mathrm{~m}$, (c) $D=0.290 \mathrm{~m}$.

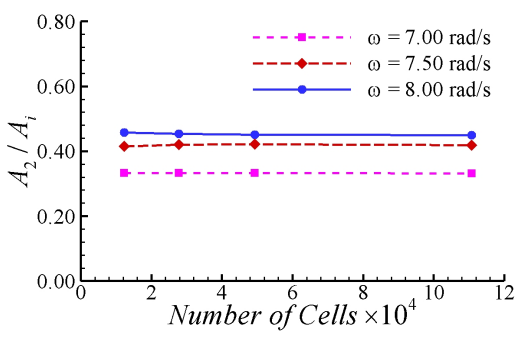

(a)

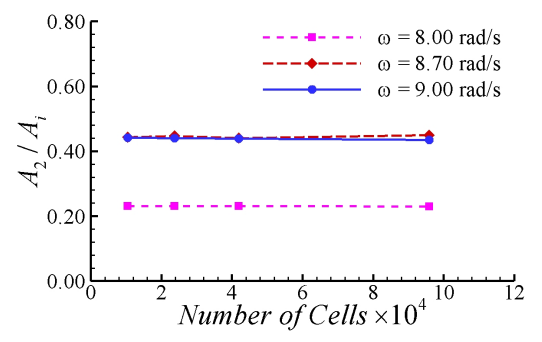

(b)

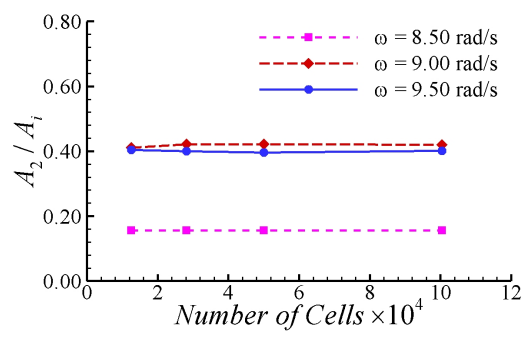

(c)

FIG. 5: Mesh convergent test for various geometries with different incident wave frequencies. (a) $D=0.094 \mathrm{~m}, B=0.150 \mathrm{~m}$, (b) $D=0.186 \mathrm{~m}, B=0.150 \mathrm{~m}$, (c) $D=0.290 \mathrm{~m}, B=0.150 \mathrm{~m}$

sloshing motion is more likely to generate the breaking phenomenon. This might be the reason for the slight discrepancy between the experimental and numerical data. However, the linear sloshing flow model only works well at non-resonant frequencies. For frequencies close to the sloshing natural frequency $\omega_{n}$, the zero sway motion amplitude and the overpredicted results are observed in the linear sloshing flow solutions. The essential assumption of the ideal fluid, irrotational flow, and linearized free surface condition is the primary reason for the inaccurate numerical results, where the exaggerated sloshing forces and the 180-degree phase at $\omega=\omega_{n}$ are generated from the linear sloshing flow model. Furthermore, the discrepancy between the present viscous flow model and the nonlinear sloshing flow model in Rognebakke and Faltinsen (2003) are also observed in Figs. 6a and 6c demonstrating the indispensability of the viscous sloshing flow model in the coupling analysis.

From the comparison and analysis, it is confirmed that the numerical coupling model can produce accurate results for sway motion responses.

\section{INFLUENCE OF INCIDENT WAVE FREQUENCY AND AMPLITUDE}

The dependence of the sway motion amplitude on the incident wave frequency and incident wave amplitude is investigated. The amplitude of the sway motion response is defined as $A_{2}$, which is computed based on the average responses in the 'steady-state stage' or 'near steady-state stage.' The validation study in Sec. III] shows that the numerical model well-reproduces the studied scenario for the coupling sloshing problem. Thus, it is employed to investigate the sway motion response induced from external waves and internal sloshing actions. The primary objective is to study the influence of the internal sloshing flow configuration, especially the nonbreaking and breaking phenomena, on the behavior of coupling actions. The 9 geometries given in Table $\mathrm{I}$ are adopted here, where the scope of incident wave frequency is from 6.0$11.0 \mathrm{rad} / \mathrm{s}$.

The explanations begin with the sway motion response from the smallest incident wave amplitude $A_{i}=0.005 \mathrm{~m}$, as shown in Fig. 7. A comparison between the results with and without the sloshing action shows the significant effect of internal sloshing on the sway motion amplitude. Two particular frequencies, lower and higher critical frequencies of $\omega_{l}$ and 


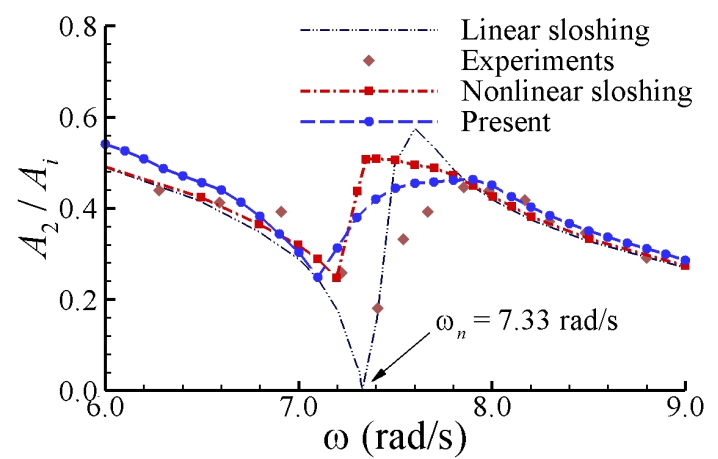

(a)

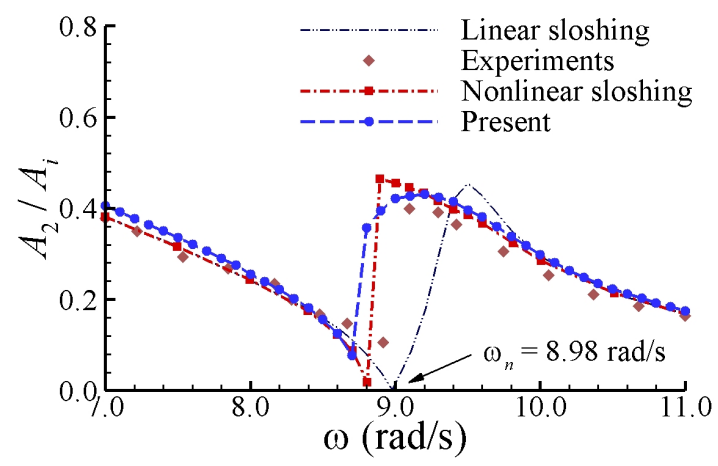

(c)

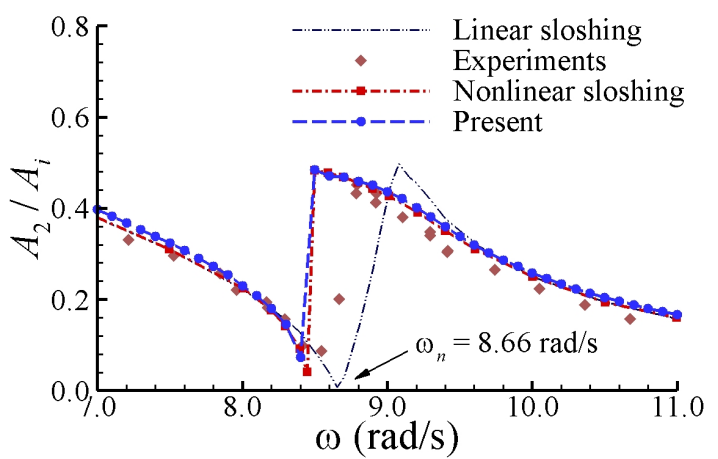

(b)

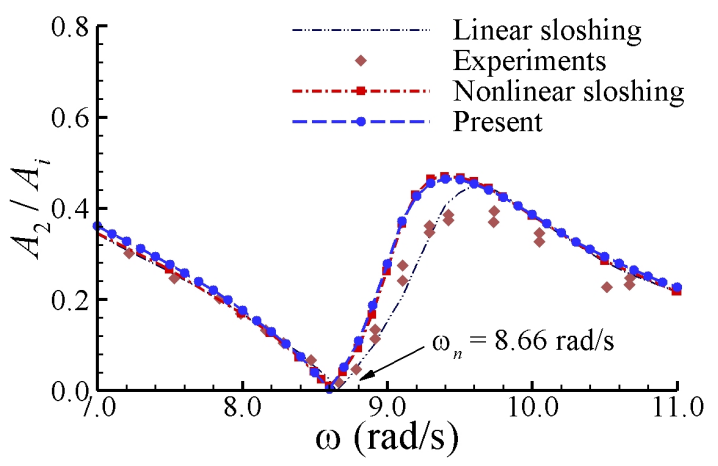

(d)

FIG. 6: Validations for the sway motion amplitudes with different filling conditions. The linear and nonlinear sloshing results in Rognebakke and Faltinsen (2003) are presented with the internal damping coefficient $\xi$ from Keulegan (1958).

(a) $B=0.150 \mathrm{~m}, D=0.094 \mathrm{~m}$, and $\xi=0.0030$, (b) $B=0.150 \mathrm{~m}, D=0.186 \mathrm{~m}$, and $\xi=0.0030$,

(c) $B=0.150 \mathrm{~m}, D=0.290 \mathrm{~m}$, and $\xi=0.0037$, (d) $B=0.300 \mathrm{~m}, D=0.186 \mathrm{~m}$, and $\xi=0.0030$.

$\omega_{h}$ corresponding to the minimal and maximal sway motion amplitudes, respectively, are defined to analyze the coupling sloshing results. The minimal and maximal values are the most significant resistant and enhanced actions of the internal sloshing flow, which are also the key physical measures of the two critical frequencies. Furthermore, a three-phase variation (low-frequency range, medium-frequency range, and highfrequency range) is suggested to describe the sway motion amplitude against the incident wave frequency. The mediumfrequency range is the region between the two critical frequencies, where a rapid increase in the sway motion amplitude with the incident wave frequency is observed. This indicates that the process of internal sloshing action changed from anti-sway motion to enhanced-sway motion. The lowand high-frequency ranges are defined as where the coupling effect has resistant and enhanced actions on the sway motion amplitude, respectively. Based on the descriptions above, it is observed that the behavior of the sway motion response around the medium-frequency range is the essential mechanism of the coupling sloshing action.

The numerical results for the sway motion amplitude at the incident wave amplitude $A_{i}=0.005 \mathrm{~m}$ suggest that the lower critical frequency $\omega_{l}$ always equals the sloshing natural fre- quency $\omega_{n}$. Meanwhile, the zero-amplitude sway motion is found at the frequency $\omega_{l}=\omega_{n}$. This is explained by the fact that the sloshing-induced internal force and the wave-induced external force are nearly out-of-phase with almost the same magnitude, as shown in Fig. 8a. Moreover, although the frequency is close to the sloshing natural frequency, the time signal of the internal sloshing force is still symmetric and appears as a sinusoidal function, implying the coupling sloshing effect is generally linear. This can also be confirmed from the agreement between the linear and viscous sloshing flow results in Fig. 7. Note that the available linear results were reported in Rognebakke and Faltinsen (2003), where only the cases of $B=$ $0.150 \mathrm{~m}$ with $D=0.094,0.186,0.290 \mathrm{~m}$ and $B=0.300 \mathrm{~m}$ with $D=0.186 \mathrm{~m}$ were presented. Furthermore, Fig. $8 \mathrm{~b}$ shows variations in the magnitude and phase shift of the internal sloshing force, which causes the rapid increase of the sway motion amplitude in the medium-frequency region. Figure $8 \mathrm{c}$ shows that the internal sloshing force has an approximately $0.44 \pi$ phase delay compared to the external wave force at the high critical frequency, which is different from the in-phase relationship. The sloshing force evolution is still symmetric and oscillates sinusoidally at the incident wave frequency. These sinusoidal characteristics in the sloshing force evolution imply they are 


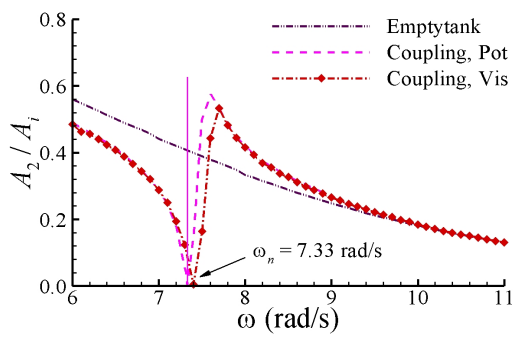

(a)

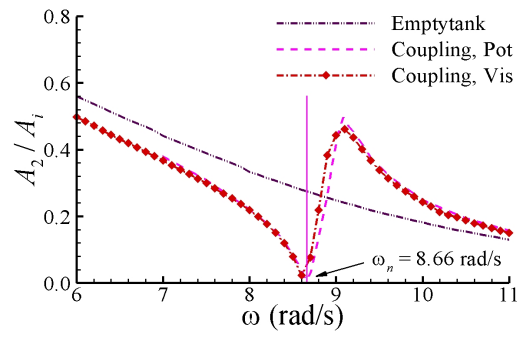

(d)

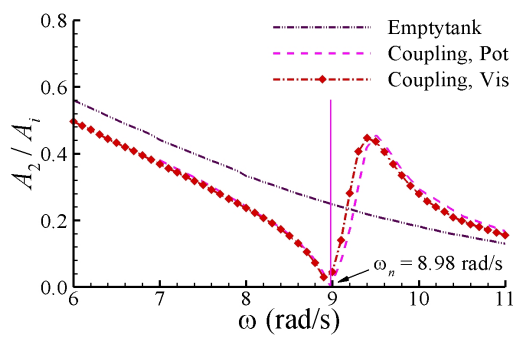

(g)

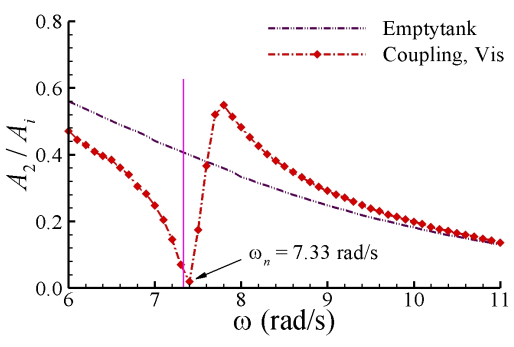

(b)

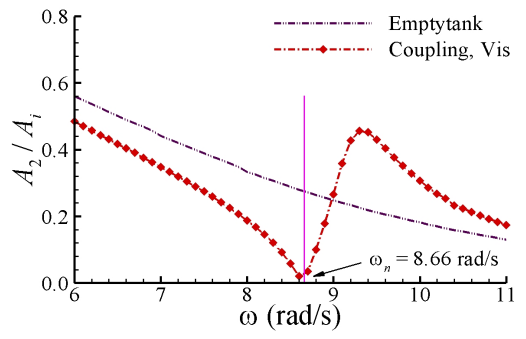

(e)

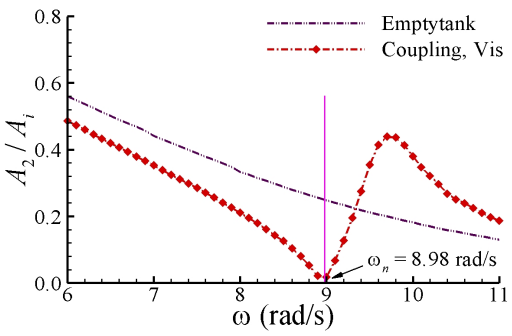

(h)

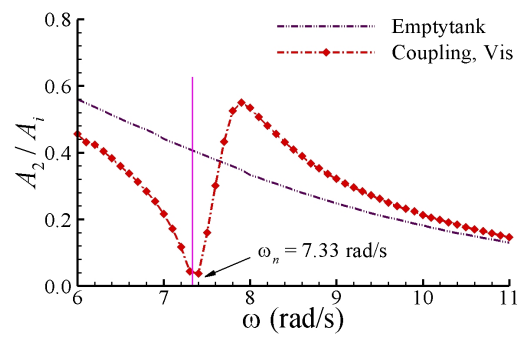

(c)

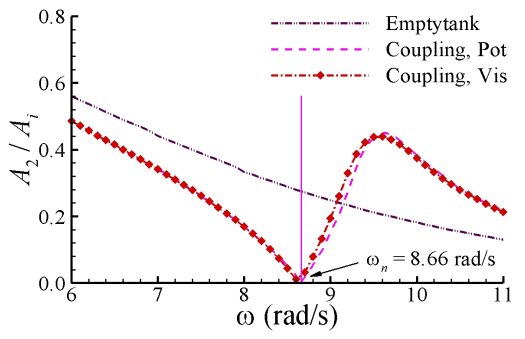

(f)

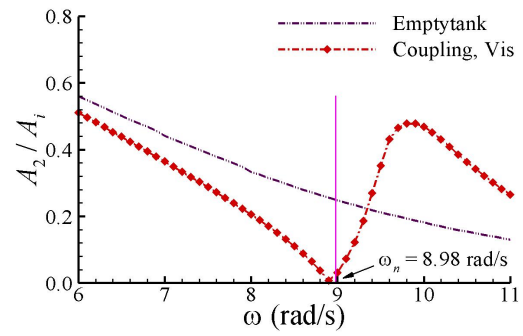

(i)

FIG. 7: Comparison of the sway motion amplitudes with and without the influence of internal sloshing flow motion at an incident wave amplitude of $A_{i}=0.005 \mathrm{~m}$.

(a) $B=0.150 \mathrm{~m}$, and $D=0.094 \mathrm{~m}$, (b) $B=0.225 \mathrm{~m}$, and $D=0.094 \mathrm{~m}$, (c) $B=0.300 \mathrm{~m}$, and $D=0.094 \mathrm{~m}$,

(d) $B=0.150 \mathrm{~m}$, and $D=0.186 \mathrm{~m}$, (e) $B=0.225 \mathrm{~m}$, and $D=0.186 \mathrm{~m}$, (f) $B=0.300 \mathrm{~m}$, and $D=0.186 \mathrm{~m}$,

(g) $B=0.150 \mathrm{~m}$, and $D=0.290 \mathrm{~m}$, (h) $B=0.225 \mathrm{~m}$, and $D=0.290 \mathrm{~m}$, (i) $B=0.300 \mathrm{~m}$, and $D=0.290 \mathrm{~m}$.

non-breaking states for internal sloshing flow. This is confirmed from the non-breaking free surface profile in the second row of Fig. 8 . Therefore, the behavior of the sway motion response under the incident wave amplitude of $A_{i}=0.005 \mathrm{~m}$ is taken as the coupling effect for the linear and non-breaking sloshing flow configurations.

To demonstrate the nonlinear characteristics of the coupling sloshing effect, special attention is given to the influence of the incident wave amplitude on the sway motion response. A wide range of incident wave amplitudes of $A_{i}$ from $0.005-$ $0.030 \mathrm{~m}$ is adopted for the simulations, as shown in Fig. 9. The numerical comparison suggests that the influence of the incident wave amplitude on the behavior of the sway motion response is described from the three-phase variation. The sway motion amplitude is nearly independent of the incident wave amplitude at the low- and high-frequency ranges, implying the minimal effect of the nonlinear coupling sloshing action. In the medium-frequency region, the sway motion response has a strong sensitivity to the incident wave amplitude. The nonlinear growth of the sloshing-induced forces with respect to the amplitude of the sloshing excitation from different incident wave amplitudes is the cause of this phenomenon. With the increased incident wave amplitude, the normalized sway motion amplitudes deviate more from the results of $A_{i}=0.005$ $\mathrm{m}$. This indicates that the nonlinear influence of the internal sloshing flow on the sway motion amplitude is more important for larger incident wave amplitudes.

Variations in the sway motion response around the mediumfrequency range also suggest that the increased incident wave amplitude not only affects the magnitude of the sway motion amplitude but also leads to the changes in the characteristic$\mathrm{s}$ of the variation for the sway motion amplitude. In this study, the sway motion response at the incident wave amplitude of $A_{i}=0.005 \mathrm{~m}$ is defined as Type1, where the zero sway motion amplitude $A_{2} / A_{i}=0$ at the frequency $\omega_{l}=\omega_{n}$ is the most important characteristic. Furthermore, Fig. 9 shows that 

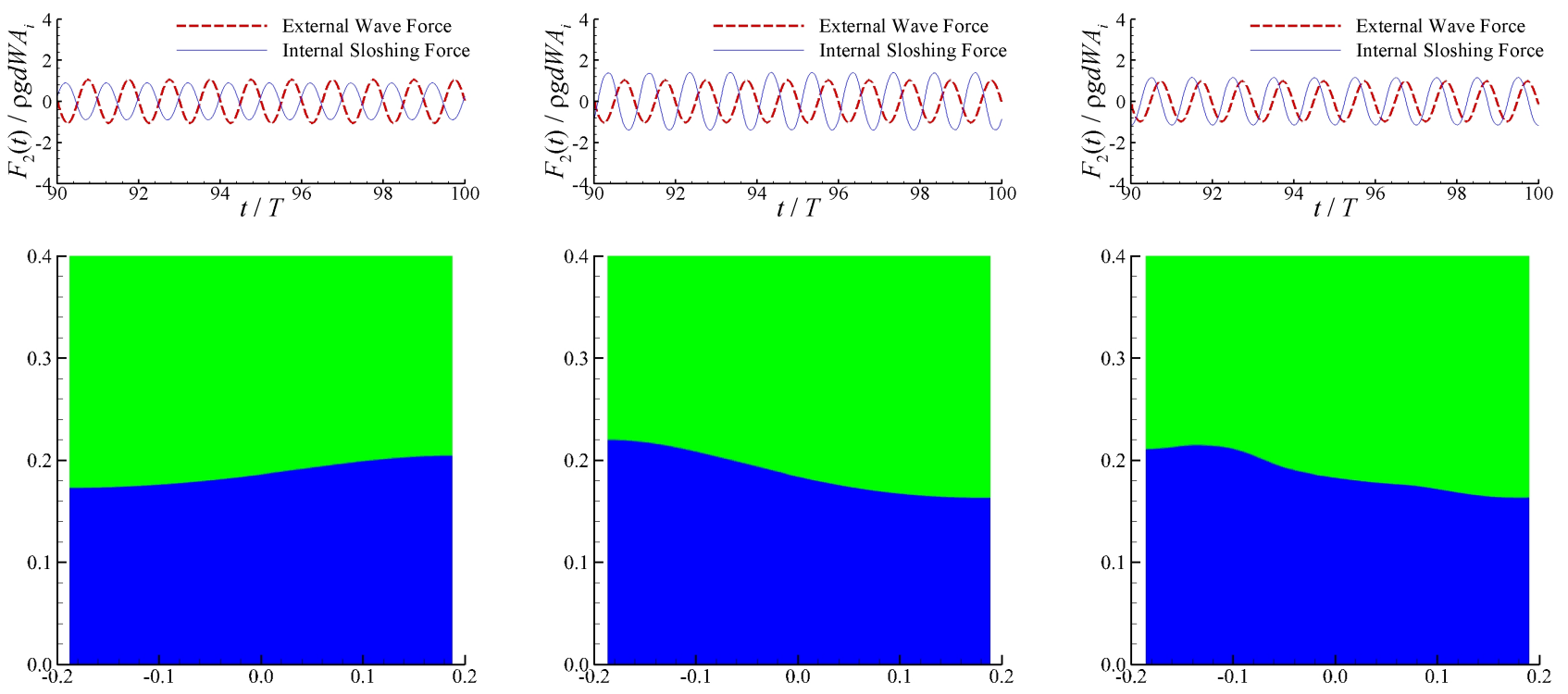

(a)

(b)

(c)

FIG. 8: Comparison of the time signals between the external wave and internal sloshing forces and the corresponding internal sloshing free surface pattern of B150D186 under $A_{i}=0.005 \mathrm{~m}\left(\omega_{l}=\omega_{n}=8.60 \mathrm{rad} / \mathrm{s}\right)$.

(a) $\omega=8.60 \mathrm{rad} / \mathrm{s}$, (b) $\omega=8.80 \mathrm{rad} / \mathrm{s}$, (c) $\omega=9.10 \mathrm{rad} / \mathrm{s}$.

the increased incident wave amplitude leads to the minimal sway motion amplitude $A_{2} / A_{i} \neq 0$ at the lower critical frequency $\omega_{l}<\omega_{n}$. In this case, the other two variation types are suggested to describe the sway motion response. The Type 2 variation shows a continuous increase from the lower critical frequency $\omega_{l}$ to the higher critical frequency $\omega_{h}$. Typical examples are cases with a filling depth of $D=0.094 \mathrm{~m}$ at the incident wave amplitude of $A_{i}=0.030 \mathrm{~m}$ in Figs. 9a $9 \mathrm{c}$. For the Type 3 variation, a sudden jump around the lower critical frequency is observed. A quintessential example is observed in Figs. 9d 9i for filling depths of $D=0.186$ and $0.290 \mathrm{~m}$ when the incident wave amplitude is $A_{i}=0.030 \mathrm{~m}$. These three types of sway motion variations are summarized in Table III.

Figures 10 and 11 show typical internal sloshing force evolutions and free surface snapshots at the time of maximal sway motion response around the lower critical frequency for the Type 2 and Type 3 variations, respectively. Distinct from the symmetric and sinusoidal characteristic in Fig. 8 for the nonbreaking variation type (Type1), impulsive behavior in the sloshing force signal is observed in these figures. The impulsive behavior suggests there is breaking sloshing flow motion in the tank, which is confirmed from the corresponding free surface profile. This indicates that the Type 2 and Type 3 variations are generated by the breaking sloshing flow action. Furthermore, evident differences are observed in the sloshing force evolutions between the Type 2 and Type 3 variations. In Fig. 10, the impulsive force and breaking sloshing flow appear in all sub-figures, indicating breaking sloshing flow occurs at all frequencies around the lower critical frequency. The sloshing force evolution patterns in the figures are relatively similar.
The major difference between them is that the external wave and sloshing internal forces have different phase shifts, which leads to a continuous increase of the sway motion amplitude for the medium-frequency range in Type2. For the Type 3 variation in Fig. 11, the sloshing force evolution shows sinusoidal and impulsive characteristics at the lower critical frequency and adjacent frequencies. Correspondingly, the internal sloshing flows at different conditions show the non-breaking and breaking states, respectively. Moreover, changes in the sloshing flow states (breaking and non-breaking) lead to sudden phase transitions for the sloshing forces and free surface profiles between adjacent frequencies. This causes a sudden jump in the variation of the sway motion amplitude around the lower critical frequency for the Type 3 variation.

The above results allow conducting a detailed analysis of the influence of the incident wave amplitude towards the sway motion response. As shown in Fig. 9 and Tab. IV, the increased incident wave amplitude can initially lead to changes in the sway motion response from the non-breaking (Type1) and breaking (Type2 and Type 3 ) types. The lower critical frequency then reduces with further increases to the incident wave amplitude, which also deviates more from the sloshing natural frequency. This indicates that a larger incident wave amplitude can more easily induce the breaking phenomenon, which implies more violent sloshing flow action during the coupling process. Furthermore, Fig. 12 shows the variations in the normalized internal sloshing forces at different incident wave amplitudes at corresponding lower critical frequencies, including a comparison with the normalized external wave force evolution. The decreased normalized internal sloshing force is observed with the increased incident wave amplitude, 


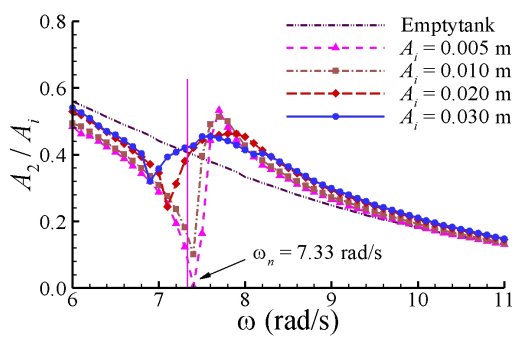

(a)

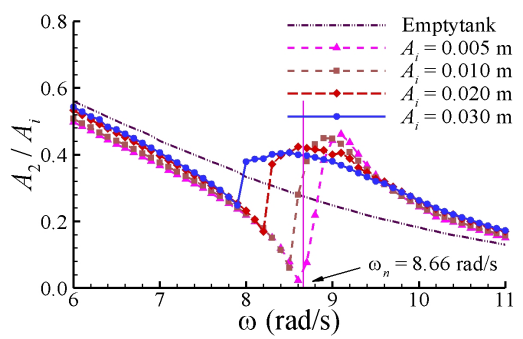

(d)

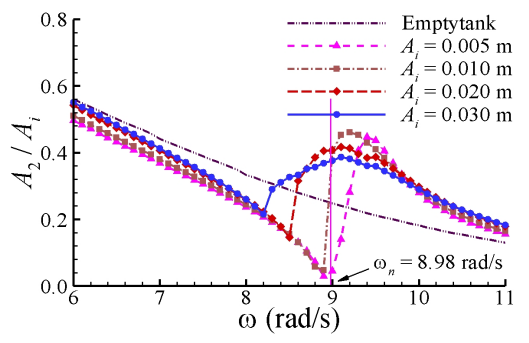

(g)

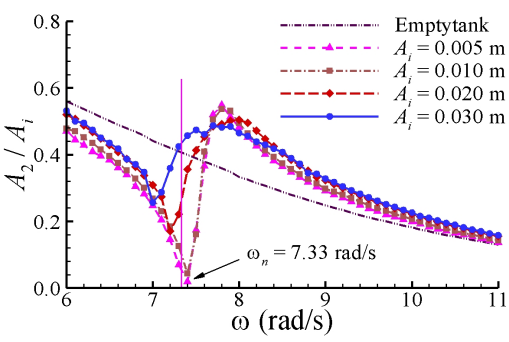

(b)

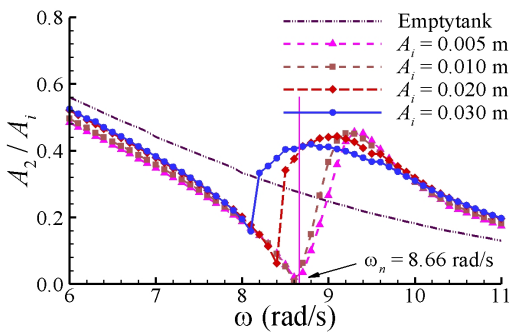

(e)

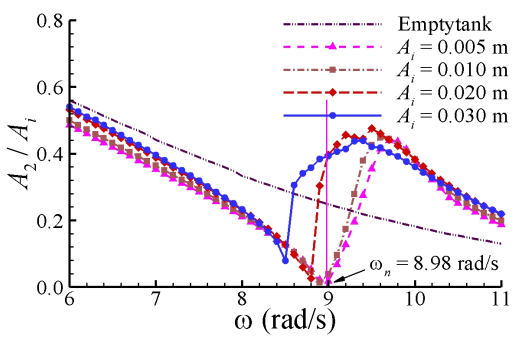

(h)

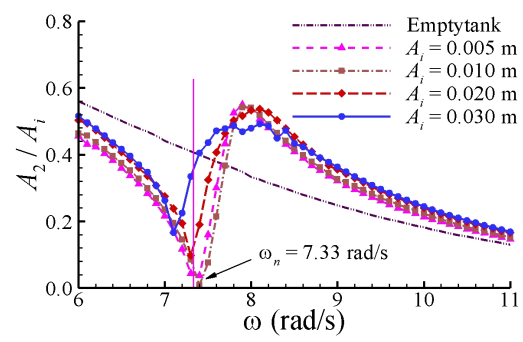

(c)

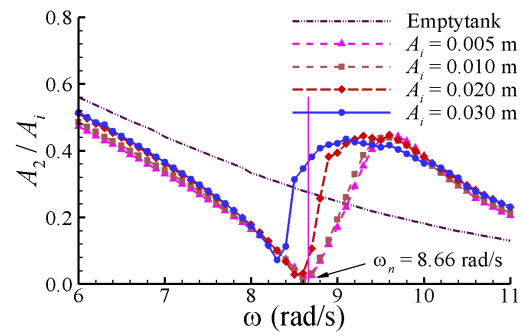

(f)

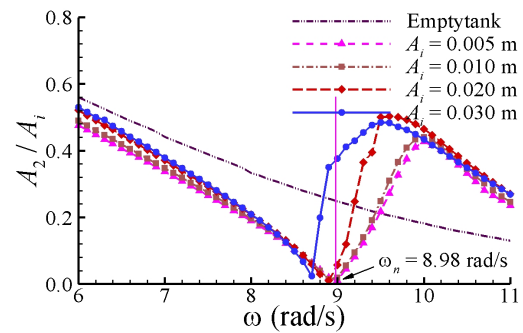

(i)

FIG. 9: Comparison of the sway motion amplitudes for various incident wave amplitudes.

(a) $B=0.150 \mathrm{~m}$, and $D=0.094 \mathrm{~m}$, (b) $B=0.225 \mathrm{~m}$, and $D=0.094 \mathrm{~m}$, (c) $B=0.300 \mathrm{~m}$, and $D=0.094 \mathrm{~m}$,

(d) $B=0.150 \mathrm{~m}$, and $D=0.186 \mathrm{~m}$, (e) $B=0.225 \mathrm{~m}$, and $D=0.186 \mathrm{~m}$, (f) $B=0.300 \mathrm{~m}$, and $D=0.186 \mathrm{~m}$,

(g) $B=0.150 \mathrm{~m}$, and $D=0.290 \mathrm{~m}$, (h) $B=0.225 \mathrm{~m}$, and $D=0.290 \mathrm{~m}$, (i) $B=0.300 \mathrm{~m}$, and $D=0.290 \mathrm{~m}$.

TABLE III: Definitions for the different variation types of sway motion responses

\begin{tabular}{lccc}
\hline Name & Frequency & Amplitude & Characteristic between the lower and higher critical frequencies \\
\hline Type1 & $\omega_{l} \approx \omega_{n}$ & $\left(A / A_{i}\right)_{\omega_{l}} \approx 0$ & Continuous increase in the medium-frequency range \\
Type2 & $\omega_{l}<\omega_{n}$ & $\left(A / A_{i}\right) \omega_{l}>0$ & Continuous increase in the medium-frequency range \\
Type3 & $\omega_{l}<\omega_{n}$ & $\left(A / A_{i}\right) \omega_{l}>0$ & Sudden jump in the medium-frequency range \\
\hline
\end{tabular}

indicating a smaller resistant action for the internal sloshing force at the lower critical frequency. This leads to an increased minimal sway motion amplitude at the lower critical frequency for larger incident wave amplitudes. This phenomenon implies that the increased incident wave amplitude decreases the internal sloshing effect when the breaking phenomenon occurs. This is confirmed from the decreased discrepancy between the results with and without the coupling sloshing effect for the different breaking types in Fig. 9. These results demonstrate that the sloshing flow configurations (i.e., nonbreaking or breaking) are the primary reason for the different types of sway motion responses. The breaking sloshing flow pattern can strengthen the nonlinear coupling sloshing effec$\mathrm{t}$ but decreases the differences in the sway motion responses with and without the sloshing action.

\section{INFLUENCE OF TANK BREADTH AND FILLING DEPTH}

The influence of the tank breadth on the sway motion response based on the incident wave condition is investigated

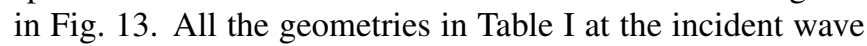
amplitudes of $A_{i}=0.005,0.010,0.020$, and $0.030 \mathrm{~m}$ are pre- 

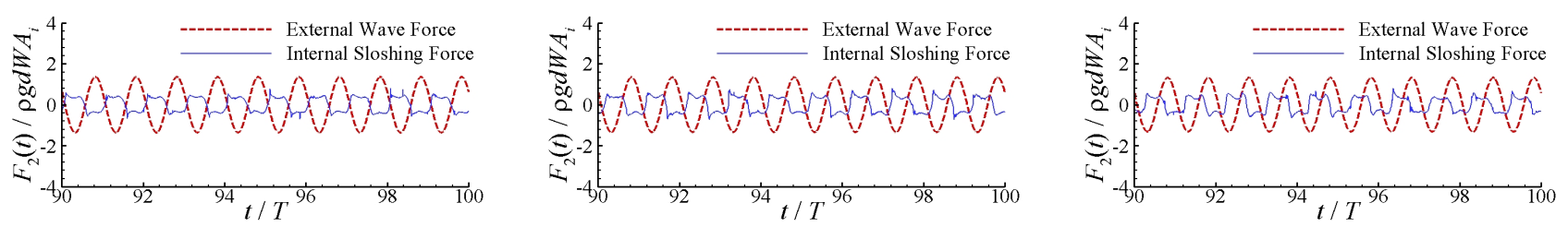

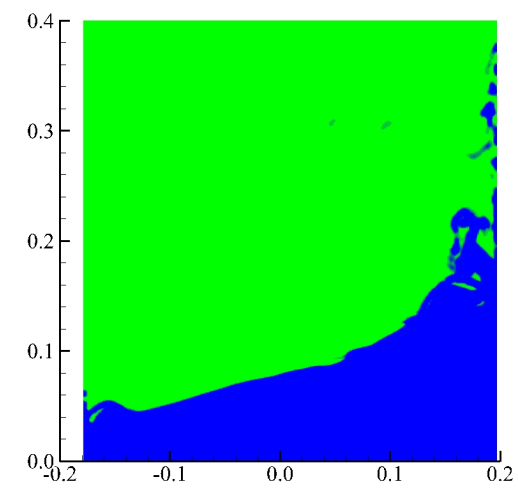

(a)

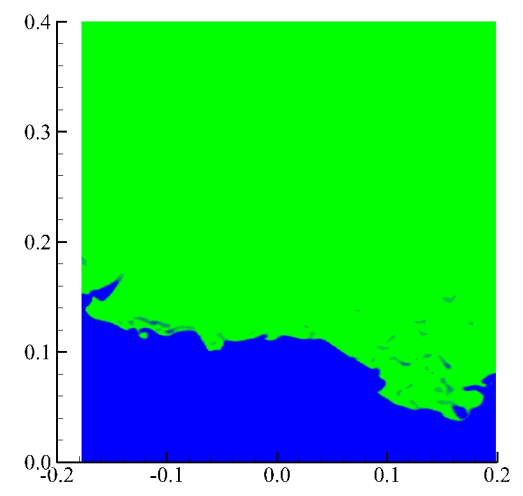

(b)

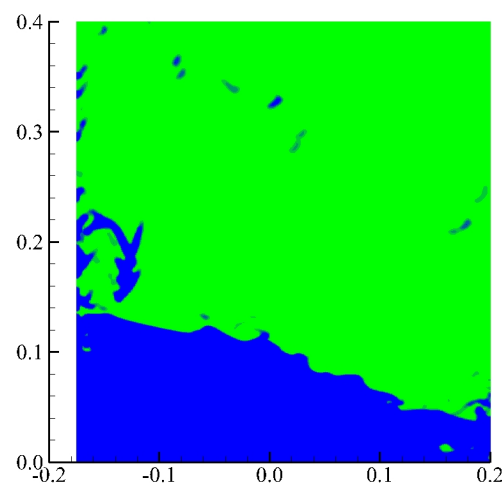

(c)

FIG. 10: Comparison of time signals between the external wave and internal sloshing forces with the corresponding internal sloshing free surface pattern for B150D094 at $A_{i}=0.030 \mathrm{~m}$ for the Type2 variation.

(a) $\omega=6.90 \mathrm{rad} / \mathrm{s}$, (b) $\omega=7.00 \mathrm{rad} / \mathrm{s}$, (c) $\omega=7.10 \mathrm{rad} / \mathrm{s}$.
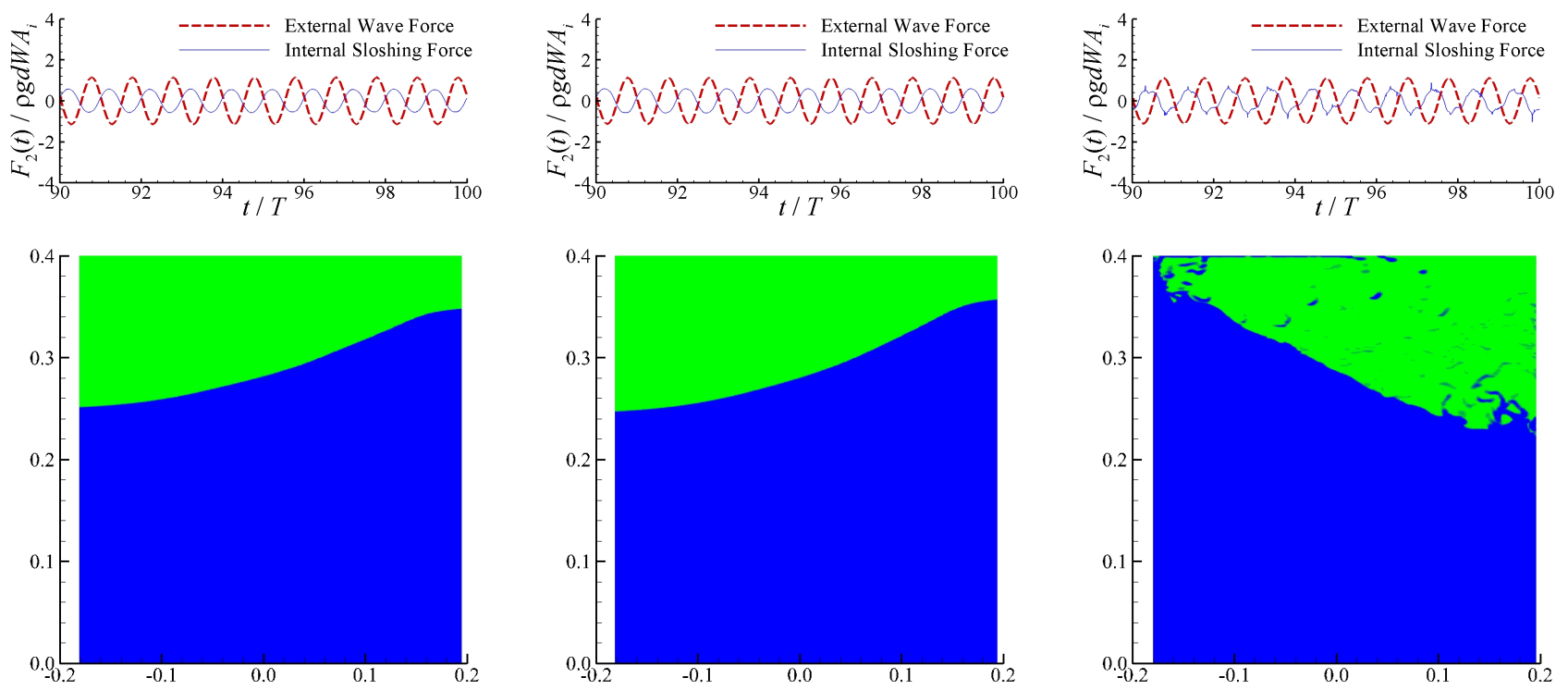

(a)

(b)

(c)

FIG. 11: Comparison of time signals between the external wave and internal sloshing forces with the corresponding internal sloshing free surface pattern for B150D290 at $A_{i}=0.030 \mathrm{~m}$ for the Type 3 variation.

(a) $\omega=8.10 \mathrm{rad} / \mathrm{s}$, (b) $\omega=8.20 \mathrm{rad} / \mathrm{s}$, (c) $\omega=8.30 \mathrm{rad} / \mathrm{s}$.

sented, including the case without internal sloshing flow action. The comparison covers the sloshing natural frequency $\omega_{n}$, which is dependent of the tank breadth. The numerical simulations show that the three-phase variation with the incident wave frequency is still suitable to describe the influence of the tank breadth. At the low- and high-frequency ranges, the sway motion amplitude decreases and increases with the increased tank breadth, reflecting the greater resistance and enhanced actions of the internal sloshing flow, respectively. In the medium-frequency range region, although the behav- 
TABLE IV: Values of the lower critical frequency in the present test cases.

\begin{tabular}{|c|c|c|c|c|c|c|}
\hline Filling Depth & Natural Frequency & Tank Breadth & Low & er Critical Fre & equency $\left(\omega_{l} / \mathrm{r}\right.$ & $\mathrm{ad} / \mathrm{s})$ \\
\hline$(D / \mathrm{m})$ & $\left(\omega_{n} / \mathrm{rad} / \mathrm{s}\right)$ & $(B / \mathrm{m})$ & $A_{i}=0.005 \mathrm{~m}$ & $A_{i}=0.010 \mathrm{~m}$ & $A_{i}=0.015 \mathrm{~m}$ & $A_{i}=0.020 \mathrm{~m}$ \\
\hline & & 0.150 & 7.40 & 7.40 & 7.10 & 6.90 \\
\hline 0.094 & 7.33 & 0.225 & 7.40 & 7.40 & 7.20 & 7.00 \\
\hline & & 0.300 & 7.40 & 7.40 & 7.30 & 7.10 \\
\hline & & 0.150 & 8.60 & 8.50 & 8.20 & 7.90 \\
\hline 0.186 & 8.66 & 0.225 & 8.60 & 8.60 & 8.40 & 8.10 \\
\hline & & 0.300 & 8.60 & 8.60 & 8.50 & 8.30 \\
\hline & & 0.150 & 8.90 & 8.90 & 8.50 & 8.20 \\
\hline 0.290 & 8.98 & 0.225 & 9.00 & 8.90 & 8.80 & 8.50 \\
\hline & & 0.300 & 9.00 & 8.90 & 8.90 & 8.70 \\
\hline
\end{tabular}

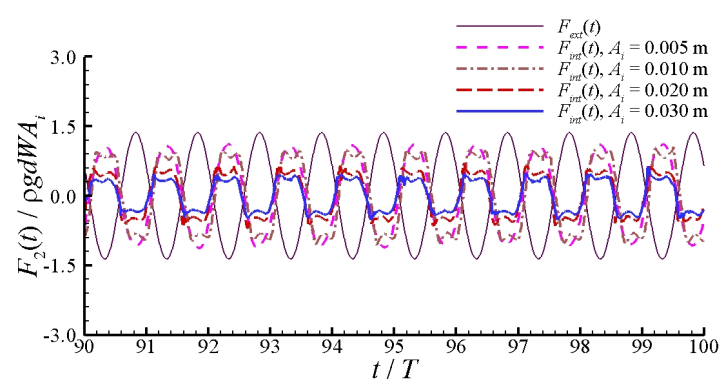

(a)

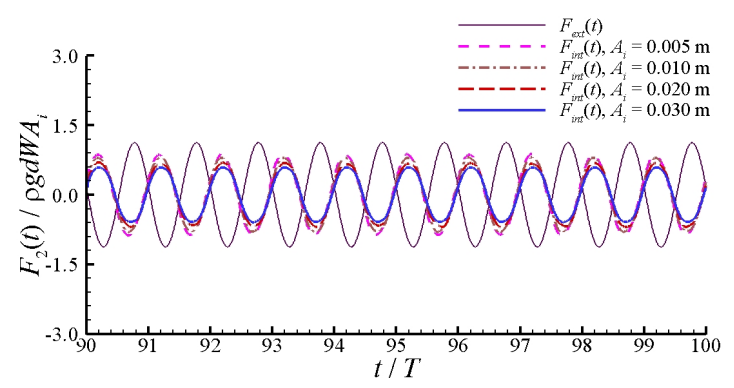

(b)

FIG. 12: Comparison of the normalized internal sloshing forces induced from various incident wave amplitudes at the lower critical frequency. (a) $B=0.150 \mathrm{~m}$, and $D=0.094 \mathrm{~m}$, (b) $B=0.150 \mathrm{~m}$, and $D=0.290 \mathrm{~m}$.

ior of the sway motion amplitude cannot be described with a simple increase or decrease variation, the general tendency of the coupling sloshing results deviate more from the empty tank results as observed with the increased tank breadth. For a specific sway motion response, the increased tank breadth can theoretically lead to a larger internal sloshing force on the tank, which has a more significant impact on the sway motion response of the box section. This also agrees with the numerical results discussed above and confirms that the increased tank breadth can strengthen the influence of the internal sloshing flow motion.

The detailed variations in the sway motion response in the medium-frequency region are considered, and the essential mechanism of the coupling sloshing action with different tank breadths is analyzed. In Figs. 13j 131, for an incident wave amplitude of $A_{i}=0.030 \mathrm{~m}$, a larger lower critical frequency and smaller minimal sway motion amplitude are observed for greater tank breadths. This is the typical behavior of the tank breadth effect on the sway motion response for breaking configurations (Type 2 and Type 3 ). The out-of-phase relationship between the internal sloshing and external wave forces at the lower critical frequency generates a stronger resistant action for the internal sloshing flow due to the larger tank breadth, which leads to a decreased sway motion amplitude. The above phenomenon is confirmed from the force evolutions in Figs. $14 \mathrm{c}$ and $14 \mathrm{~d}$, including the increased internal sloshing force with a larger tank breadth and the outof-phase relationship between the internal sloshing and exter- nal wave forces. Therefore, a larger tank breadth can generate the same internal sloshing force with a smaller sloshing flow. The smaller sloshing amplitude makes it more difficult for the breaking sloshing phenomenon to occur, which also leads to the lower critical frequency increases and gives rise to the sloshing natural frequency. In Figs. $13 \mathrm{a}, 13 \mathrm{c}$ for $A_{i}=$ $0.005 \mathrm{~m}$, the results of $A_{2} / A_{i}=0$ and $\omega_{l}=\omega_{n}$ are unaffected by changes in the tank breadth. As the relatively small sway motion amplitude at the frequency $\omega_{l}=\omega_{n}$ can lead to extreme internal sloshing forces from the non-breaking sloshing flow, the same magnitude and 180-degree phase difference between the internal sloshing and external wave forces are unaffected by further increases to the tank breadth, as shown in Fig. 14a Therefore, a larger tank breadth only retains the zero-amplitude sway motion. The other typical behavior of the tank breadth effect on the sway motion response for the non-breaking type (Type1) is where the tank breadth effect leads primarily to an increased higher critical frequency. For some other cases, the combined behavior of the non-breaking and breaking types is observed. As an example, Figs. $13 \mathrm{~d}$ 13f show that an increased tank breadth can change the sway motion response from the breaking type to the non-breaking type, which then behaves as non-breaking. This is confirmed from the variations in the sloshing force evolution with the tank breadth in Fig. 14b, where the non-sinusoidal and sinusoidal characteristics are respectively observed for $B=0.150$ $\mathrm{m}$ and all other breadths. In Fig. 15, the sloshing-free surface shows a breaking pattern for a tank breadth of $B=0.150$ 


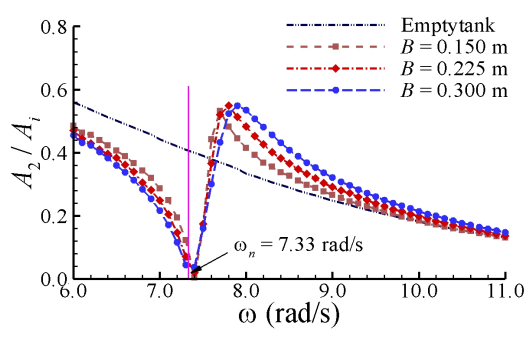

(a)

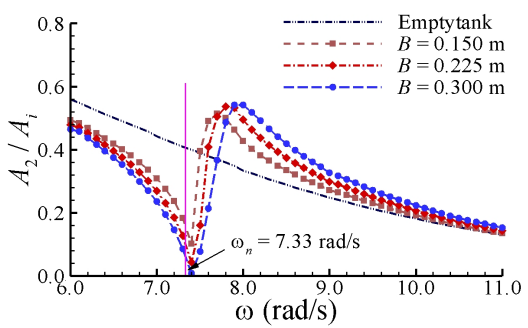

(d)

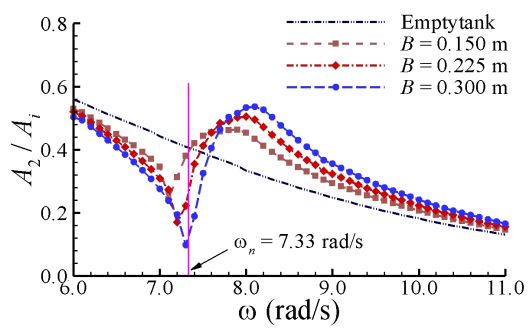

$(\mathrm{g})$

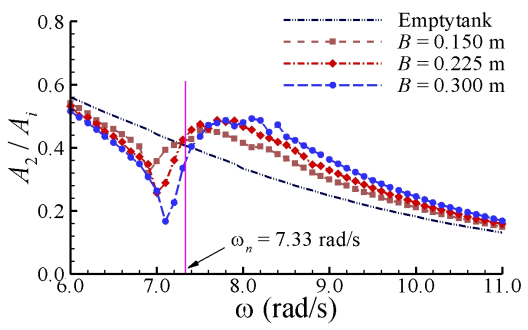

(j)

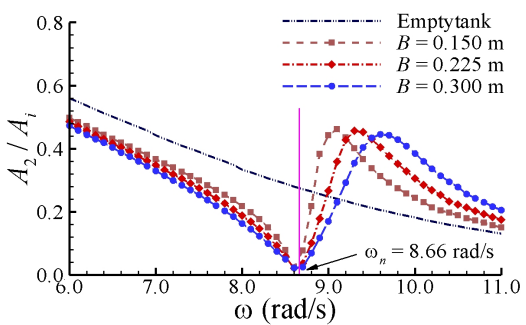

(b)

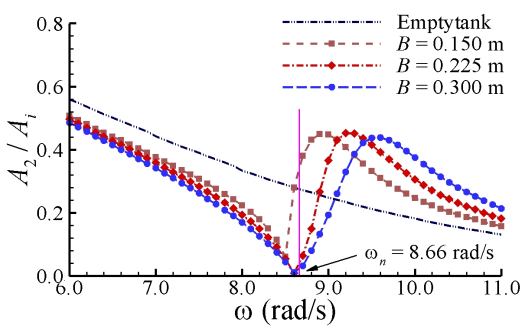

(e)

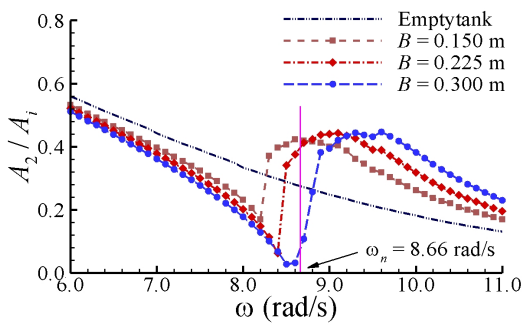

(h)

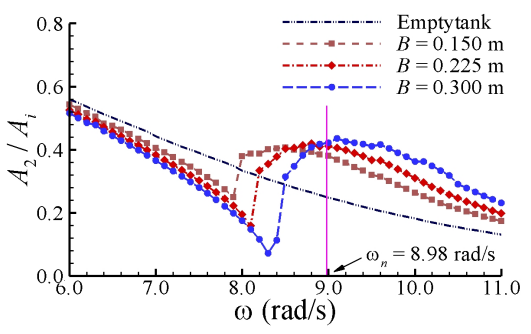

(k)

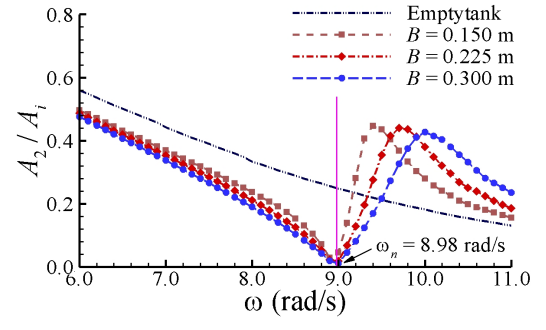

(c)

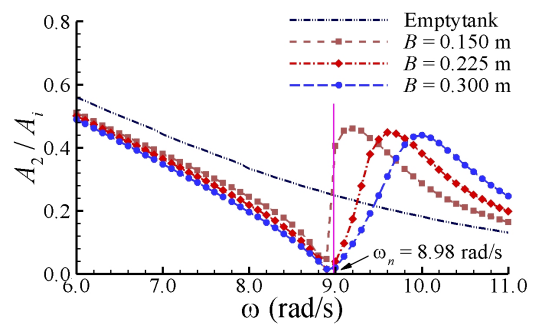

(f)

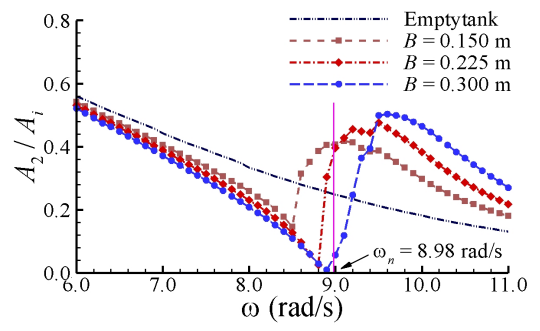

(i)

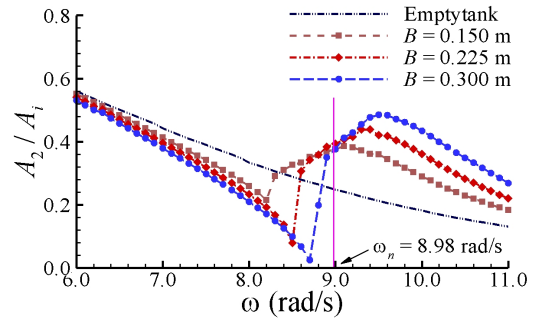

(1)

FIG. 13: Comparison of the sway motion amplitudes for various tank breadths.

(a) $D=0.094 \mathrm{~m}$, and $A_{i}=0.005 \mathrm{~m}$, (b) $D=0.186 \mathrm{~m}$, and $A_{i}=0.005 \mathrm{~m}$, (c) $D=0.290 \mathrm{~m}$, and $A_{i}=0.005 \mathrm{~m}$,

(d) $D=0.094 \mathrm{~m}$, and $A_{i}=0.010 \mathrm{~m}$, (e) $D=0.186 \mathrm{~m}$, and $A_{i}=0.010 \mathrm{~m}$, (f) $D=0.290 \mathrm{~m}$, and $A_{i}=0.010 \mathrm{~m}$,

(g) $D=0.094 \mathrm{~m}$, and $A_{i}=0.020 \mathrm{~m}$, (h) $D=0.186 \mathrm{~m}$, and $A_{i}=0.020 \mathrm{~m}$, (i) $D=0.290 \mathrm{~m}$, and $A_{i}=0.020 \mathrm{~m}$,

(j) $D=0.094 \mathrm{~m}$, and $A_{i}=0.030 \mathrm{~m}$, (k) $D=0.186 \mathrm{~m}$, and $A_{i}=0.030 \mathrm{~m}$, (l) $D=0.290 \mathrm{~m}$, and $A_{i}=0.030 \mathrm{~m}$.

$\mathrm{m}$, while the flow patterns for $B=0.225$ and $0.300 \mathrm{~m}$ only show the non-breaking free surface profiles. This is a complete change from the breaking type to the non-breaking type with the increased tank breadth, which confirms that a larger tank breadth suppresses violent internal sloshing motions during coupling actions.

Figure 16 compares the sway motion responses between d- ifferent filling depths. Distinct from the tank breadth effec$t$, greater filling depths not only increase the water mass in the sway-motion-system but also increase the sloshing natural frequency $\omega_{n}$. Numerical simulations suggest that the lower and higher critical frequencies $\omega_{l}$ and $\omega_{h}$ have the same trend with the sloshing natural frequency $\omega_{n}$, indicating violent sloshing motion is the essential factor for the coupling slosh- 


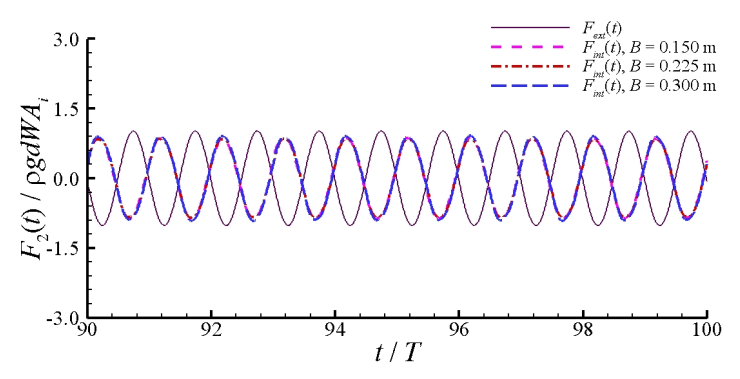

(a)

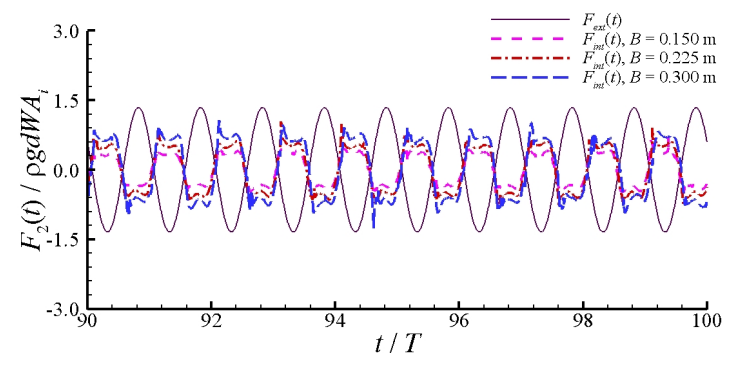

(c)

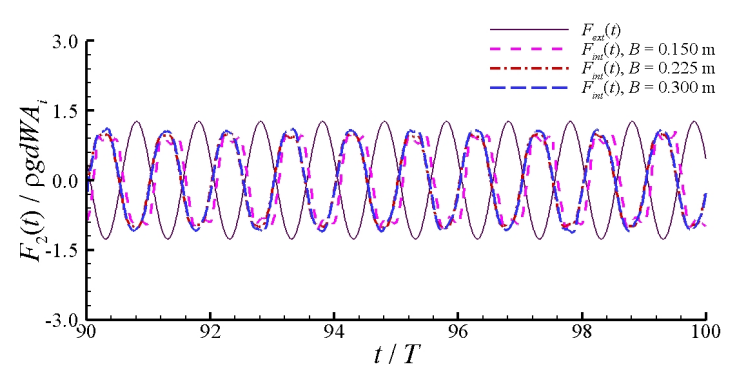

(b)

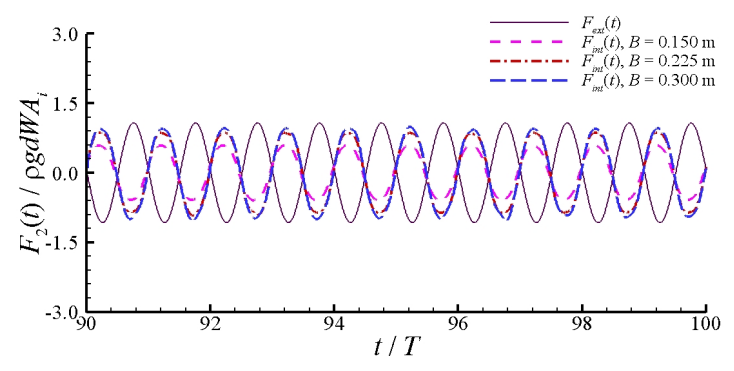

(d)

FIG. 14: Comparison of the normalized internal sloshing forces with various tank breadths at the lower critical frequency.

(a) $D=0.290 \mathrm{~m}$, and $A_{i}=0.005 \mathrm{~m}$, (b) $D=0.094 \mathrm{~m}$, and $A_{i}=0.010 \mathrm{~m}$,

(c) $D=0.094 \mathrm{~m}$, and $A_{i}=0.030 \mathrm{~m}$, (d) $D=0.290 \mathrm{~m}$, and $A_{i}=0.030 \mathrm{~m}$.

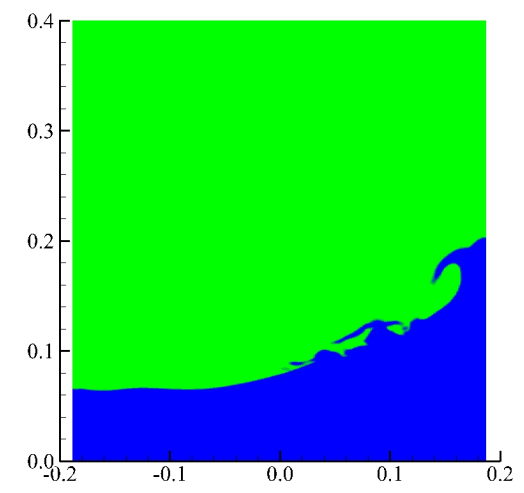

(a)

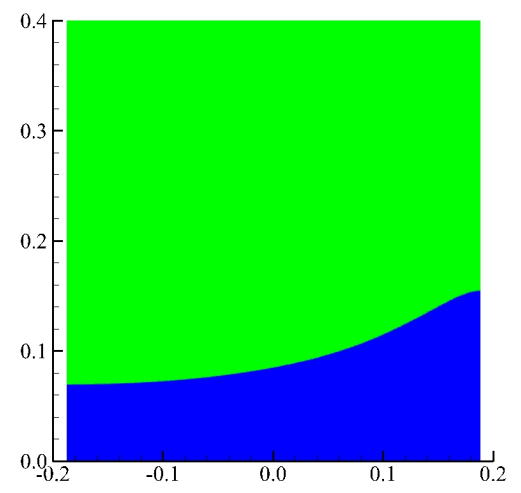

(b)

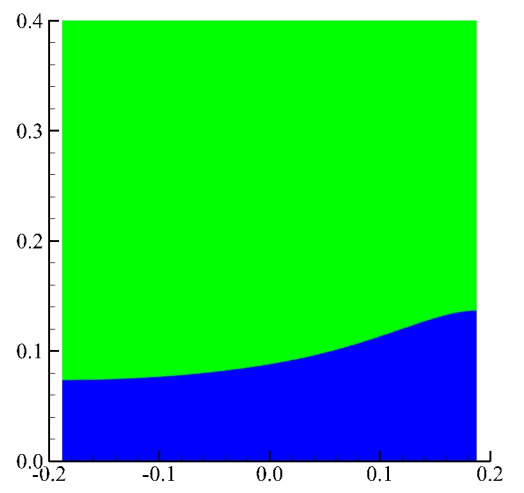

(c)

FIG. 15: Internal sloshing-free surface patterns for a filling depth of $D=0.094 \mathrm{~m}$ at the lower critical frequency under the incident wave amplitude of $A_{i}=0.010 \mathrm{~m}$ for different tank breadths. (a) $B=0.150 \mathrm{~m}$, (b) $B=0.225 \mathrm{~m}$, (c) $B=0.300 \mathrm{~m}$.

ing effect. The most significant difference in sway motion amplitudes between different filing depths is in the mediumfrequency region. In general, variations in the sway motion amplitude between $D=0.186$ and $0.290 \mathrm{~m}$ have similar characteristics, while there are differences observed for $D=0.094$ $\mathrm{m}$. As a demonstration, the breaking type of the sway motion response shows the characteristics of Type 3 for $D=0.186$ and $0.290 \mathrm{~m}$ but is Type 2 for $D=0.094 \mathrm{~m}$. The shallow filling depth of $D=0.094 \mathrm{~m}$ induces nonlinear sloshing flow motion and is the primary cause of these differences, which implies that the influence of the filling depth is complex, in addition to its influence on the natural frequency and water mass.

\section{CONCLUSION}

Numerical simulations analyze the sway motion response of a box section with internal liquid sloshing. The viscous two-phase flow model with a VOF-interface-capturing technique based on the OpenFOAM ${ }^{\circledR}$ package is established 


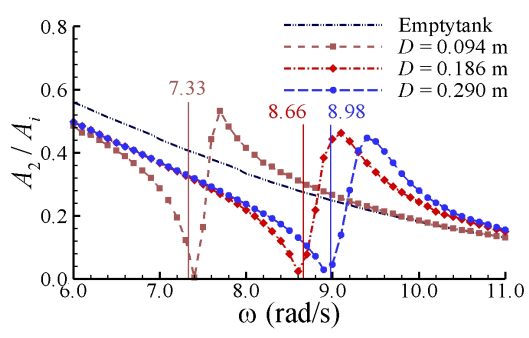

(a)

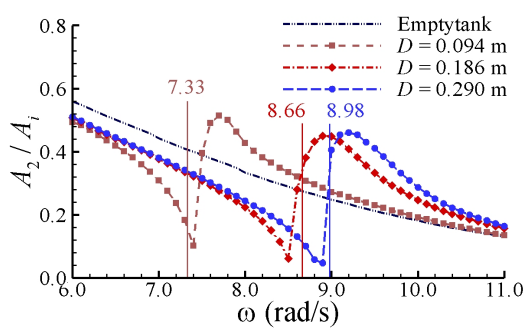

(d)

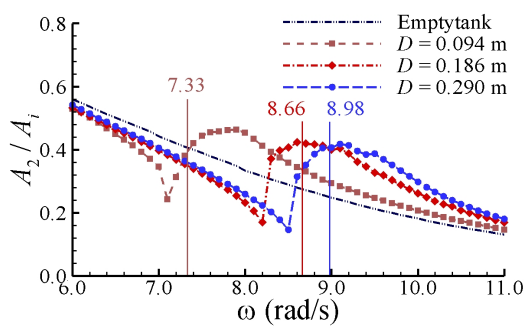

(g)

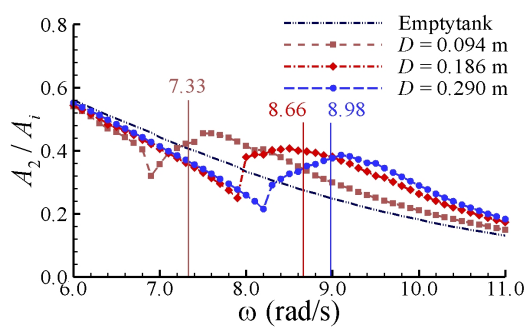

(j)

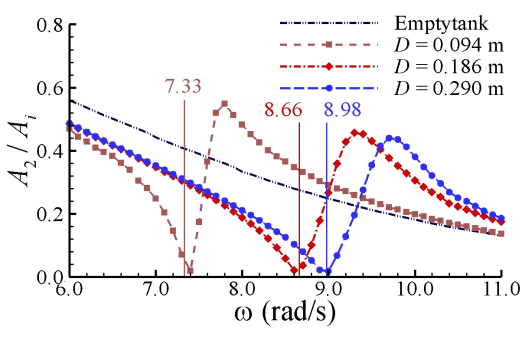

(b)

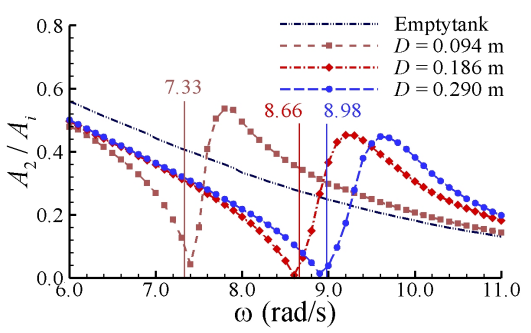

(e)

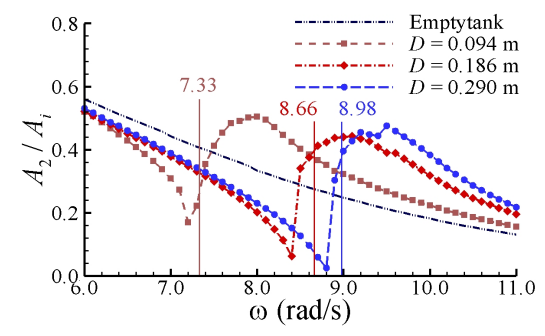

(h)

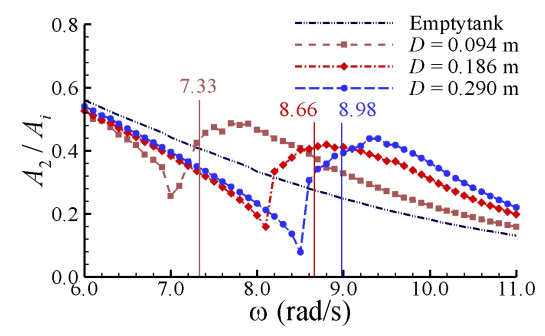

(k)

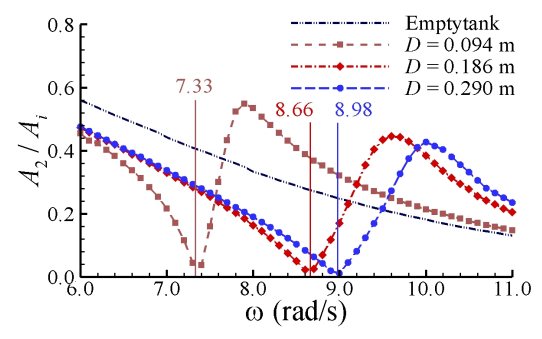

(c)

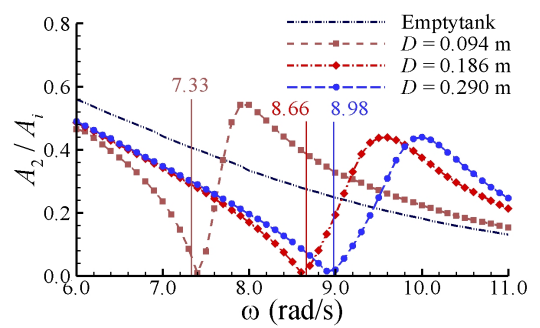

(f)

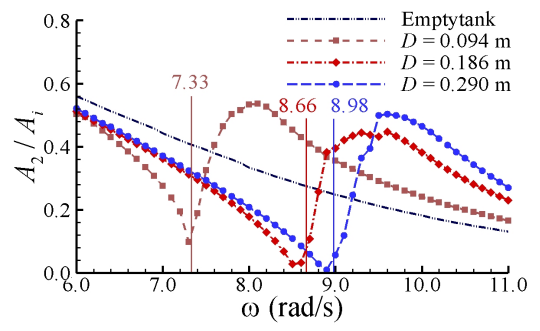

(i)

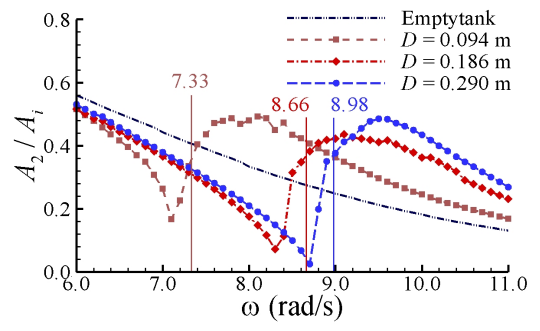

(1)

FIG. 16: Comparison of sway motion amplitudes for various filling depths.

(a) $B=0.150 \mathrm{~m}$, and $A_{i}=0.005 \mathrm{~m}$, (b) $B=0.225 \mathrm{~m}$, and $A_{i}=0.005 \mathrm{~m}$, (c) $B=0.300 \mathrm{~m}$, and $A_{i}=0.005 \mathrm{~m}$,

(d) $B=0.150 \mathrm{~m}$, and $A_{i}=0.010 \mathrm{~m}$, (e) $B=0.225 \mathrm{~m}$, and $A_{i}=0.010 \mathrm{~m}$, (f) $B=0.300 \mathrm{~m}$, and $A_{i}=0.010 \mathrm{~m}$,

(g) $B=0.150 \mathrm{~m}$, and $A_{i}=0.020 \mathrm{~m}$, (h) $B=0.225 \mathrm{~m}$, and $A_{i}=0.020 \mathrm{~m}$, (i) $B=0.300 \mathrm{~m}$, and $A_{i}=0.020 \mathrm{~m}$,

(j) $B=0.150 \mathrm{~m}$, and $A_{i}=0.030 \mathrm{~m}$, (k) $B=0.225 \mathrm{~m}$, and $A_{i}=0.030 \mathrm{~m}$, (l) $B=0.300 \mathrm{~m}$, and $A_{i}=0.030 \mathrm{~m}$.

for the internal sloshing flow, while the impulse-responsefunction (IRF) method is employed for the external ship response. To better understand the influence of wave conditions and tank configurations on the sway motion response, four incident wave amplitudes with three tank breadths and three filling depths under different wave frequencies are considered. The essential mechanism of the coupling action between the sway motion and internal sloshing is explained from the perspective of the internal sloshing flow pattern, especially for non-breaking or breaking phenomena. The main findings are illustrated as follows:

1) Two important frequencies, the sloshing natural frequency and the lower critical frequency, are recommended to understand the essential mechanism of coupling sloshing 
problems. The sloshing natural frequency is the inherent property of the sloshing flow motion, which is independent of the coupling action. The lower critical frequency corresponds to the minimal sway motion amplitude, where the out-of-phase relationship between the internal sloshing and external wave forces are observed. The physical essence of the lower critical frequency is the resistant action of the coupling sloshing as it approaches the maximum. This is generated from the coupling action between the external wave and the internal sloshing flow.

2) The lower critical frequency coincides with the sloshing natural frequency when the internal sloshing flow does not break at small incident wave amplitudes. As there is no breaking phenomenon in this case, the extreme internal sloshing force at the sloshing natural frequency can be excited by the small sway motion. Therefore, the steady-state internal sloshing force readily oscillates at the same magnitude as the external wave force, which does not cause sway motion at that frequency. Thus, the linear sloshing flow model well predicts the sway motion response, which implies the insignificant effect of the sloshing nonlinearity. This non-breaking sloshing-induced sway motion response is called the non-breaking type, or Type1, variation.

3) When the breaking phenomenon occurs, the internal sloshing force is smaller than the external wave force, which causes a non-zero sway motion amplitude at the lower critical frequency. In addition, the breaking phenomenon also generates a phase transition for the internal sloshing motion, which further affects the phase shift of the internal sloshing force and causes the lower critical frequency to deviate from the sloshing natural frequency. Therefore, the minimal sway motion amplitude and the lower critical frequency increase and decrease, respectively, with the increased incident wave amplitude. That is, the sway motion response deviates more from the linear sloshing results, which indicates the nonlinearity that results from the breaking sloshing effect becomes more important. However, differences in the sway motion response between the coupling sloshing tank and the empty tank tend to be smal1, suggesting a smaller coupling effect on the sway motion response.

4) The breaking-sloshing-induced sway motion response has two variation types, denoted as Type 2 and Type 3 . Type 2 is where the sway motion amplitude has a rapid continuous increase from the lower critical frequency to the higher incident frequency, while Type 3 is where the amplitude has a sudden jump around the lower critical frequency. The difference between the two breaking types is primarily due to the steady-state sloshing flow patterns at frequencies around the lower critical frequency. The breaking sloshing phenomenon is observed at all frequencies for Type2. A similar sloshing flow configuration generates continuous phase shift variations in the internal sloshing force, which continuously varies the sway motion amplitude. For Type 3 , the non-breaking and breaking sloshing patterns are found in the lower critical frequency and its adjacent frequencies, respectively. A saltation of the phase transition appears between the adjacent frequencies, leading to a sudden jump in the sway motion amplitude.

5) The increased tank breadth can generate crucial coupling action for the internal sloshing flow on the sway motion response, which is confirmed by the increased difference in the sway motion amplitudes between the cases with and without sloshing flow. This is caused by the increased internal sloshing force from the larger tank breadth. However, the increased tank breadth causes the lower critical frequency to approach to the sloshing natural frequency, where the minimal sway motion amplitude tends to be small. Therefore, the sway motion response tends to approach the non-breaking type from the breaking type, which implies that nonlinear behaviors of the coupling action weaken for larger tank breadths.

\section{ACKNOWLEDGMENTS}

This work was supported by the National Natural Science Foundation of China with Grants No. 51909027. The first author acknowledges the Supercomputer Center of Dalian University of Technology for providing computing resources.

\section{DATA AVAILABILITY STATEMENT}

All data generated or analyzed during this study are included in this article.

\section{REFERENCES}

Bouscasse, B., Colagrossi, A., Soutoiglesias, A., and Cercospita, J. L., "Mechanical energy dissipation induced by sloshing and wave breaking in a fully coupled angular motion system. I. theoretical formulation and numerical investigation," Physics of Fluids 26, 033103 (2014a).

Bouscasse, B., Colagrossi, A., Soutoiglesias, A., and Cercospita, J. L., "Mechanical energy dissipation induced by sloshing and wave breaking in a fully coupled angular motion system. II. experimental investigation," Physics of Fluids 26, 033104 (2014b).

Cao, X. Y., Tao, L., Zhang, A. M., and Ming, F. R., "Smoothed particle hydrodynamics (SPH) model for coupled analysis of a damaged ship with internal sloshing in beam seas," Physics of Fluids 31, 032103 (2019).

Cercos-Pita, J. L., Bulian, G., Pérez-Rojas, L., and Francescutto, A., "Coupled simulation of nonlinear ship motions and a free surface tank," Ocean Engineering 120, 281-288 (2016).

Cummins, W. E., "The impulse response function and ship motions," Tech. Rep. (David Taylor Model Basin Washington DC, 1962).

Du, Y., Wang, C., and Zhang, N., "Numerical simulation on coupled ship motions with nonlinear sloshing," Ocean Engineering 178, 493-500 (2019).

Gou, Y., Kim, Y., and Kim, T.-Y., "A numerical study on coupling between ship motions and sloshing in frequency and time domains," in Proceedings of the 21th International Offshore and Polar Engineering Conference (2011) pp. 158-164.

Hirt, C. W. and Nichols, B. D., "Volume of fluid (VOF) method for the dynamics of free boundaries," Journal of Computational Physics 39, 201-225 (1981).

Huang, S., Duan, W., Han, X., Nicoll, R., You, Y., and Sheng, S., "Nonlinear analysis of sloshing and floating body coupled motion in the timedomain," Ocean Engineering 164, 350-366 (2018). 
Issa, R. I., "Solution of the implicitly discretised fluid flow equations by operator-splitting," Journal of Computational Physics 62, 40-65 (1986).

Jasak, H., Error analysis and estimation for the finite volume method with applications to fluid flows, Ph.D. thesis, Imperial College London (1996).

Jiang, S.-C., Teng, B., Bai, W., and Gou, Y., "Numerical simulation of coupling effect between ship motion and liquid sloshing under wave action," Ocean Engineering 108, 140-154 (2015).

Keulegan, G. H., "Energy dissipation in standing waves in rectangular basins," Journal of Fluid Mechanics 6, 33-50 (1958).

Kim, Y., "A numerical study on sloshing flows coupled with ship motion the anti-rolling tank problem," Journal of Ship Research 46, 52-62 (2002).

Lee, S. J. and Kim, M. H., "The effects of inner-liquid motion on LNG vessel responses," Journal of Offshore Mechanics and Arctic Engineering 132, 021101 (2010)

Li, Y. L., Su, M., Li, H., Deng, R., Wang, K. P., and Hu, Z., "Numerical research on time domain ship motions coupled with sloshing at different liquid levels and forward speeds," Ocean Engineering 178, 246-259 (2019).

Lu, L., Jiang, S., Zhao, M., and Tang, G., "Two-dimensional viscous numerical simulation of liquid sloshing in rectangular tank with/without baffles and comparison with potential flow solutions," Ocean Engineering 108, 662-677 (2015).

Malenica, S., Zalar, M., and Chen, X. B., "Dynamic coupling of seakeeping and sloshing," in Proceedings of the 13th International Offshore and Polar Engineering Conference (2003) pp. 486-492.

Mitra, S., Wang, C. Z., Reddy, J. N., and Khoo, B. C., "A 3D fully coupled analysis of nonlinear sloshing and ship motion," Ocean Engineering 39, $1-13$ (2012).

Molin, B., "LNG-FPSO's: frequency domain, coupled analysis of support and liquid cargo motion," in Proceedings of the IMAM conference, Rethymnon, Greece, 2002 (2002).

Nam, B.-W., Kim, Y., Kim, D.-W., and Kim, Y.-S., "Experimental and numerical studies on ship motion responses coupled with sloshing in waves," Journal of Ship Research 53, 68-82 (2009).
Newman, J. N., "Wave effects on vessels with internal tanks," in Proceedings of the 20th Workshop on Water Waves and Floating Bodies, Spitsbergen, Norway (2005).

Rognebakke, O. F. and Faltinsen, O. M., "Coupling of sloshing and ship motions," Journal of Ship Research 47, 208-221 (2003).

Rusche, H., Computational fluid dynamics of dispersed two-phase flows at high phase fractions, Ph.D. thesis, Imperial College London (2003).

Saripilli, J. R. and Sen, D., "Numerical studies on effects of slosh coupling on ship motions and derived slosh loads," Applied Ocean Research 76, 71-87 (2018).

Sauret, A., Boulogne, F., Cappello, J., Dressaire, E., and Stone, H. A., "Damping of liquid sloshing by foams," Physics of Fluids 27, 022103022103 (2015).

Serván-Camas, B., Cercós-Pita, J., Colom-Cobb, J., García-Espinosa, J., and Souto-Iglesias, A., "Time domain simulation of coupled sloshingseakeeping problems by SPH-FEM coupling," Ocean Engineering 123, 383-396 (2016).

$\mathrm{Su}, \mathrm{Y}$. and Liu, Z. Y., "Coupling effects of barge motion and sloshing," Ocean Engineering 140, 352-360 (2017).

Teng, B. and Eatock Taylor, R., "New higher-order boundary element methods for wave diffraction/radiation," Applied Ocean Research 17, 71-77 (1995).

Wang, J., Sun, S.-L., and Hu, J., "The coupling analysis of tank motion and sloshing by a fully nonlinear decoupling method," Nonlinear Dynamics 89, 971-985 (2017).

Zhao, D., Hu, Z., Chen, G., Chen, X., and Feng, X., "Coupling analysis between vessel motion and internal nonlinear sloshing for FLNG applications," Journal of Fluids and Structures 76, 431-453 (2018).

Zhao, W., Yang, J., Hu, Z., and Tao, L., "Coupled analysis of nonlinear sloshing and ship motions," Applied Ocean Research 47, 85-97 (2014).

Zhuang, Y. and Wan, D., "Numerical study on ship motion fully coupled with LNG tank sloshing in CFD method," International Journal of Computational Methods 16, 1840022 (2019). 